\title{
Adaptive inverse ray-tracing for accurate and efficient modeling of cross beam energy transfer in hydrodynamics simulations
}

\author{
A. Colaïtis, ${ }^{1}$ R. K. Follett, ${ }^{2}$ J. P. Palastro, ${ }^{2}$ I. Igumenschev, ${ }^{2}$ and V. Goncharov ${ }^{2}$ \\ ${ }^{1)}$ Centre Lasers Intenses et Applications, UMR 5107, 351 Cours de la libération, 33400 Talence, \\ France \\ ${ }^{2)}$ Laboratory for Laser Energetics, 250 East River Rd, Rochester, NY 14623-1212
}

(Dated: 1 July 2019)

\begin{abstract}
Integrated hydrodynamics simulations of inertial confinement fusion rely on reduced physics models. To reproduce experimental trends, these models often feature tuning parameters, but this comes with a risk: over-tuning of one model can hide physics inadequacies in another. Ray-based models of cross-beam-energy transfer (CBET) represents one of these risks. Here we present an accurate and efficient model of CBET suitable for inline implementation in 3D hydrodynamics simulations. Inverse Ray Tracing (IRT) is used to compute the ray field in a 3D permittivity profile described on an unstructured tetrahedral mesh using the IFRIIT framework (Inline Field Reconstruction and Interaction using Inverse Tracing). CBET is accounted for through perturbations to the permittivity associated with ion acoustic waves driven by the overlapped fields. Large gradients in the permittivity are resolved by coupling the IRT to a recursive Adaptive Mesh Refinement (AMR) algorithm. The use of AMR also allows for the resolution of caustics, with accurate field reconstruction performed using the Etalon integral method. Comparisons of the model with wave-based solutions from the Laser Plasma Simulation Environment (LPSE) demonstrate its ability to control energy conservation and gain convergence through AMR depth only, without the use of ad hoc physical models or artificial tuning parameters.
\end{abstract}

\section{INTRODUCTION}

Interaction of long-pulse high-power laser beams with targets entail a variety of coupling processes. In the case of High Energy Density (HED) studies, and notably direct-drive and indirect-drive Inertial Confinement Fusion (ICF) targets, the laser light couples non-linearly to electron and ion plasma modes ${ }^{1}$. These Laser Plasma Instabilities (LPI), result of microscopic-scale couplings, can grow in space and time to levels such that the fluidscale target dynamics are impacted ${ }^{2}$. This is routinely observed in the cases of low-mode asymmetry induced by light-scattering LPIs (mainly Cross-Beam Energy Transfer $(\mathrm{CBET}))^{3-6}$, or modification of shock properties through preheat from LPI-generated hot electrons ${ }^{7-9}$.

Studying the detailed dynamics of these microscopic processes requires the use of microscopic-scale models, such as Particle-in-Cell codes, electromagnetic wave solvers or even Vlasov codes. These models rely on highly detailed descriptions of light propagation processes and plasma wave physics. Such approaches are not compatible with scales larger than, at most, a few tens of micrometers over more than a few tens of picoseconds in 3-D geometries. However, since these couplings often affect the global energy balance significantly, they must be accounted for in fluid radiative-hydrodynamic codes. While LPIs have often been mimicked in these codes through various numerical parameters, notably by varying electron thermal transport flux-limiters (see e.g. Refs. 10 and 11), these do not correctly reproduce the detailed effect of LPIs on plasma dynamics. Notably, energy scattered due to Brillouin backscatter and/or CBET, often removed at the lens in hydrodynamic simulations ${ }^{6}$, may redistribute laser energy in the coronal plasma, inducing hydrodynamic perturbations and modifying other LPIs.

CBET occurs in different regimes in directly and indirectly-driven targets. In indirect drive, laser fields overlap at the laser entrance hole in almost the same region of space. As such, there is little refraction, the coupling occurs mostly between incident fields and away from caustics. However, fields are subject to polarization rotation through $\mathrm{CBET}^{12}$, kinetic effects related to the overlap of many IAWs may occur and electrons depart from Maxwellian distributions due to Langdon effect $^{13}$. In direct-drive, refraction is much more prominent, CBET occurs at large scales and contain caustic fields, and beams interact with themselves after being reflected onto caustics. As such, an ideal and unified fluid-scale descriptions of CBET for ICF should be robust with respect to refraction, describe the small scales of caustics while remaining valid there and describe laser fields in order to be able to account for polarization rotation effects.

In the last decade, many inline CBET models have emerged $^{5,6,11,14,15}$. These rely on (i) the description of the Ion Acoustic Wave (IAW) response, often using either linear fluid or linear kinetic approaches ${ }^{1,16}$, and (ii), the description of the laser intensity distribution in plasma, rendered difficult by limitations of the Geometrical Optics (GO) framework. In most hydrodynamic codes, the beam intensity is described through the proxy of grid cells, from ray powers. As such, they rely heavily on the number of rays in each cell ${ }^{17}$. A typical number required for convergence being in the 15 -30 rays per cells range for CBET calculations, which makes them less suited to high refraction regions. In addition, they do not produce physical results at laser caustics. This is caused by the underlying estimator diverging (or converges to an incor- 
rect solution) and by the mesh grid not being adapted to these fine scales. Finally, since they describe beam intensity, they are not directly suited for polarization rotation modeling.

This limited description of fields has severe consequences on the predictive capabilities of CBET models. Depending on the geometries, they are known to depend either on multipliers, swelling factors, or highlyunphysical clamping of IAW amplitudes ${ }^{4,18-20}$. While these discrepancies may be, in part, related to missing physics in IAW models or inaccurate plasma parameters in the hydrodynamics simulations, the underlying issues of the propagation models themselves must be addressed first. In a previous paper, we introduced the IFRIIT inline laser propagation model ${ }^{21}$, that aims at resolving some of these issues. Here, we describe a CBET model formulated for the IFRIIT framework, and in particular for the challenging configurations encountered in direct and indirect-drive.

The paper is organized as follows. First, the framework of the CBET studies considered in the paper is given in Sec. II. The subset of CBET physics challenges tackled here is presented in Sec. II A, while the reference code used for comparisons, $L P S E^{22,23}$, is briefly summarized in Sec. II B. Then, a description of the models implemented in the IFRIIT code is given in Sec. III. We then present an AMR method interfaced within the IRT framework of IFRIIT in Sec. IV. We highlight how this adaptive meshing notably enables the resolution of strong gradients in laser quantities, such as at sharp CBET resonances or caustics. The CBET model is presented in Sec. V. In the framework of IFRIIT, this is a formulation compatible with multi-sheeted covered spaces. Implications to the calculation of backscattered light are presented, with comparisons to LPSE. Combination of the CBET model and AMR are presented in Sec. VI, demonstrating the capability to control energy conservation and local and global CBET gains through refinement depth. Applications to direct-drive-like ICF configurations and comparisons to LPSE are presented in VII. Finally, conclusions are presented in Sec. VIII.

\section{FRAMEWORK FOR THE CBET PHYSICS STUDIES}

Overlapping laser fields in plasmas create beat waves whose ponderomotive potential drives density perturbations. When the frequency of these perturbations is close to the plasma acoustic frequency, they may parametrically grow to large amplitudes. These perturbations act like a grating for the laser light, and cause energy to be scattered in the directions of the laser fields. Due to the phase velocity of the grating itself, originating from the frequency mismatch of the lasers in the plasma frame, the light scattering is asymmetric, such that some laser fields gain energy, while others loose energy. Note that beams of the same frequency can still exchange energy when the plasma frame is in motion, which may introduce an adequate mismatch through Doppler shift. In addition, a frequency mismatch may originate from a shift of the light frequency onto time-varying density gradients ${ }^{24}$.

\section{A. CBET issues relevant to ICF and corresponding test cases}

A large variety of physics processes contribute to CBET in ICF configurations, rendering its modeling complex. These processes notably include: the linear kinetic IAW response of the plasma, the non-linear IAW response (including IAW saturation), depletion of the pump field, short-scalelength and long-scalelength plasma inhomogeneities, interaction of a beam with itself (referred to as self-CBET), feedback through ponderomotive non-linearity, effect of light frequency shift onto time-varying density profiles, interaction at sharp field boundaries (e.g. caustics), resonance broadening due to finite beam f-number, statistical effect of speckles, time-dependent behavior of the interaction, polarization rotation and phase-front deviation effects. At fluid scales, modeling these processes translates into significant challenges due to the constrains on CPU-efficiency of propagation models. As an example, the quasi-converged 2-D simulations with the wave-solver LPSE presented at the end of Sec. VII (which do not capture all of the processes listed above), required 30 cells per wavelength and took approximately 300k CPU hours for 8 ps of simulation time.

In this paper, we aim to model the interaction in steady-state and in 3-D, with linear kinetic and linear fluid IAW response, pump depletion, long-scalelength inhomogeneities and sharp field features. In addition, we tackle the difficulties related to caustics, where the ray optics framework breaks down, and high refraction rates, which are difficult to model accurately. We note that the effects of frequency shift onto time-varying density profiles $^{24}$ and gain broadening due to finite f-number are implemented but not used nor described here. In addition, we mention that speckle statistics and polarization rotation effects will be considered in future work.

The examples tackled in this paper, shown in Fig. 1, are formulated with increasing complexity. First, selfCBET is studied in a 1-D configuration, which includes strong pump-depletion and caustic fields (Fig. 1(a)). This is relevant to any interaction at low incidence angle in presence of a reflected field. While the CBET is enabled here through a velocity profile, we note that the frequency shift of the light near the critical density, estimated of the order of $1 \stackrel{A}{ }$, will enable this interaction too. The plasma velocity profile is typical of a lasercreated plasma: increasing away from the target and directed outward. It reaches zero velocity at the ablation front where it changes direction. Then, we study sharp field boundary effects on CBET in a homogeneous plasma configuration, as illustrated in Fig. 1(b). Essentially, sharp features, such as laser caustics or steep 
(a)

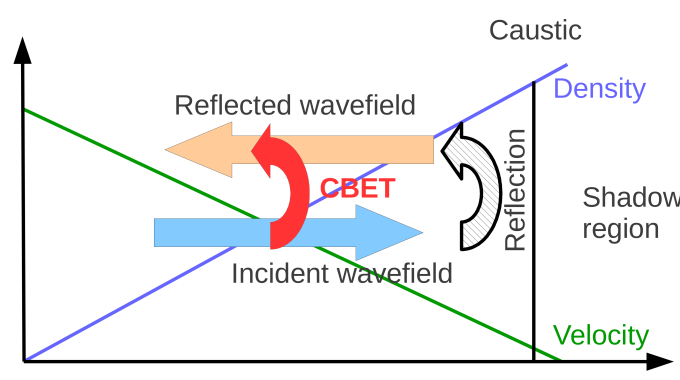

(c)

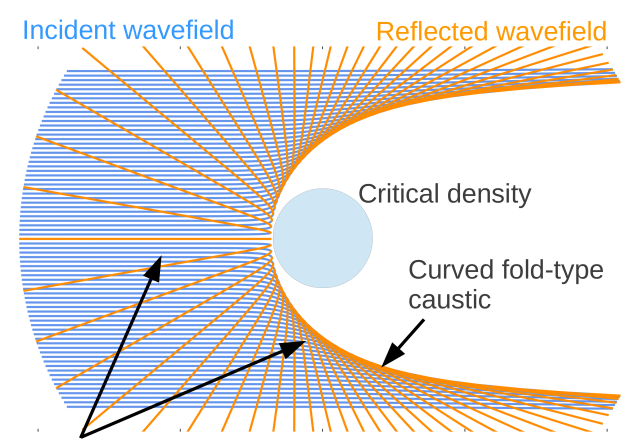

Self-CBET interaction (b)

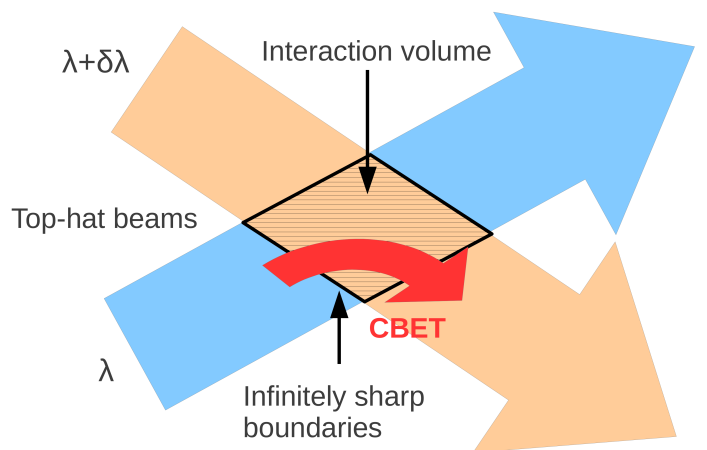

(d)

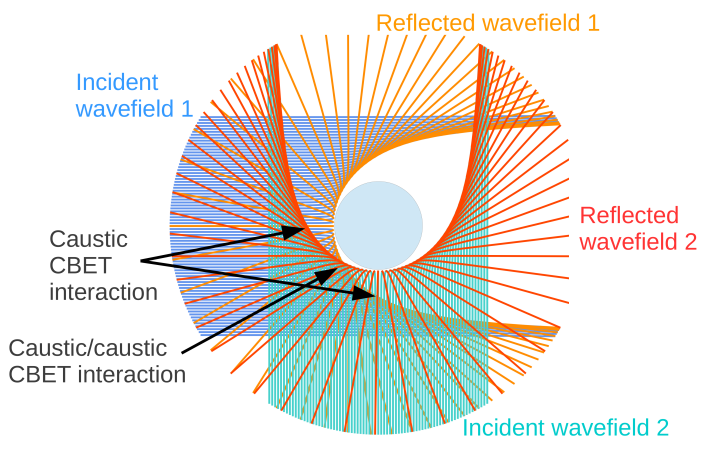

FIG. 1. Summary of the CBET physics test cases considered in this paper. (a) A wavefield incident onto a caustic pumps the reflected wavefield in a velocity and density profile. (b) Two beams exchange energy with infinitely sharp edges in a constant density plasma. (c) A beam interacts with itself in a spherical density profile, in high refraction rate regions and at a caustic. (d) Four-wave mixing in a spherical density profile with two beams, combining self-CBET at high refraction rate, caustic-wave CBET interaction and caustic-caustic CBET interaction.

interaction boundaries, will imprint mesh perturbations on CBET gains through aliasing, and lead to decrease of energy conservation properties. This effect is rarely considered in calculations. Third, we study a direct-drive relevant case, of a beam incident onto a spherical density and velocity profile (see Fig. 1(c)). This case combines the self-CBET of case (a), sharp fields of case (b), high refraction rates, and challenges the accuracy of caustic field reconstruction models where some of the interaction takes place. Finally, the same case is conducted with two beams, i.e. four-wave mixing CBET in spherical geometry, adding CBET interaction occurring purely at the caustics of the two beams (see Fig. 1(d)).

\section{B. LPSE}

Throughout the paper, we present comparisons between the ray-based code IFRIIT and the wave-based reference code LPSE. LPSE, for Laser Plasma Simulation Environment, is a platform for studying the nonlinear laser-plasma interaction at fluid scales, in regimes relevant to ICF. In LPSE, the time-dependent laser light propagation is described by the time-enveloped Maxwell equations. The plasma is a fixed inhomogeneous background, with low-frequency density and velocity perturbations obtained from the linearized fluid equations, in- cluding the ponderomotive modifications due to the beating of laser waves. In the calculations, a constant Landau damping rate of the IAWs is prescribed, as well as a constant Inverse Bremsstrahlung damping rate at the critical density (noted $\nu_{\mathrm{IB}}^{*}$ in subsequent sections). Full details about the code are given in Ref. 22 .

\section{THE IFRIIT FRAMEWORK}

Current inline models implemented in hydrodynamics codes rely on laser intensity estimators, that use rays powers to define intensity onto the hydrodynamic grid. This is done either by (i) binning rays in cells, e.g. statistical ${ }^{6}$ or Monte-Carlo approaches ${ }^{25}$, (ii) dividing the ray power by the ratio of the ray path-length to the cell volume ${ }^{15}$, (iii) dividing the ray power by the area formed from adjacent rays ${ }^{14}$, or (iv) integrating envelope intensity fields of Gaussian rays onto cells ${ }^{5}$. The formulation of a new approach to inline propagation was motivated by the poor convergence properties of these methods in high refraction region, the lack of correctness at caustics, the lack of capabilities to resolve laser-relevant scales instead of hydrodynamic-relevant scales, and their predicting of laser intensities and not fields. Here, we describe the framework of the IFRIIT inline model, which was formulated to overcome these limitations. 


\section{A. The IFRIIT code}

The IFRIIT code $^{21}$ (Inline Field Reconstruction and Interaction using Inverse Tracing) is a module for computing propagation and nonlinear interaction of laser light with plasmas, at large scales and with performance compatible with the requirements of inline modeling. The model combines Inverse Ray Tracing (IRT) techniques, real-valued and Complex-valued Geometrical Optics (GO/CGO) with Etalon Integrals (EI), and Adaptive Mesh Refinement (AMR) algorithms. The combination of the first two techniques allows for the efficient and accurate computation of laser fields in 3 -D, including at caustics of Fold type, and without any limiters or artificial coefficients. The CGO ray-trace engine allows for accurate modeling of diffraction processes and wavefield refraction on the imaginary part of the dielectric permittivity. Finally, AMR, introduced in the present paper, allows for the resolution of multiple spatial scales to enhance the accuracy of LPI computations. In this paper, we focus on the GO approximation implemented in IFRIIT, i.e. we neglect wave refraction on the imaginary part of the CBET coupling coefficient. Simple estimates show that this is a good approximation for CBET, where the IAW amplitude typically saturates through nonlinear processes below a few percent ${ }^{1,12}$.

\section{B. Inverse Ray Tracing}

Contrary to conventional ray-trace algorithms employed in hydrodynamic codes, IRT consists in finding the phase space ray parameters that connect rays from an initial ray surface (e.g. a lens) to so-called "observation points" in a medium. Employing IRT avoids many pitfalls of conventional rigid-scale estimation techniques. Notably, it removes the dependency of the field computation on the number of rays per cells, and guarantees that every ray contributing to the field at a given point is accounted for. Furthermore, IRT enables accurate ray phase and amplitude computation since no averaging process is employed, thus providing a robust method to compute ray fields at any point in the medium.

The phase space ray parameters involved in IRT are: (i) $\left(\zeta_{1}, \zeta_{2}\right) \in \mathbb{C}$, that parametrize the complex-valued initial ray position $\mathbf{r}$ and momentum $\mathbf{p}$ on the initial ray surface through functions $\mathbf{R}_{0}\left(\zeta_{1}, \zeta_{2}\right)$ and $\mathbf{P}_{0}\left(\zeta_{1}, \zeta_{2}\right)$, and (ii) a complex-valued arc-length parameter $\tau$, involved in the ray trajectory equations:

$$
\begin{array}{r}
\mathbf{r}\left(\zeta_{1}, \zeta_{2}, \tau\right)=\mathbf{R}_{0}\left(\zeta_{1}, \zeta_{2}\right)+\int_{0}^{\tau} \mathbf{p}\left(\zeta_{1}, \zeta_{2}, \tilde{\tau}\right) \mathrm{d} \tilde{\tau} \\
\mathbf{p}\left(\zeta_{1}, \zeta_{2}, \tau\right)=\mathbf{P}_{0}\left(\zeta_{1}, \zeta_{2}\right)+\frac{1}{2} \int_{0}^{\tau} \nabla \epsilon\left(\mathbf{r}\left(\zeta_{1}, \zeta_{2}, \tilde{\tau}\right)\right) \mathrm{d} \tilde{\tau}
\end{array}
$$

with $\epsilon$ the dielectric permittivity of the medium.

When constructing the mapping $\left(\zeta_{1}, \zeta_{2}, \tau\right) \rightarrow(x, y, z)$, one may find several solutions per beam for a given observation point. Discontinuities in the number of solutions in space are called caustics, and are located in real space for GO. At caustics, the Jacobian of the associated coordinate transform from phase space to configuration space becomes non-invertible, which leads to the well know divergence of the ray amplitude term of the ray-optics framework (see Eq. (4)). The set of ray parameters constituting the mapping $\left(\zeta_{1}, \zeta_{2}, \tau\right) \rightarrow(x, y, z)$ such that the associated rays go through the same number of caustics is called a ray sheet ${ }^{31}$. As such, ray sheets, noted $\Omega$, are bijective mappings from $\left(\zeta_{1}, \zeta_{2}, \tau\right)$ to $(x, y, z)$, where the Jacobian of the associated coordinate transform is smooth in space. In GO, different ray sheets are separated by caustics.

\section{The Geometrical Optics ray field}

We recall the formulation of the ray field in the GO framework. The wavefield at a given observation point is obtained by summing the contribution from all rays reaching that observation point. The field amplitude $|u|_{\text {GO }}$ of an individual GO ray reads:

$$
\begin{array}{r}
|u|_{\mathrm{GO}}=\left|A_{0}(\tau)\right| \exp \left(\imath k_{0} \psi^{(1)}(\tau)\right) \\
A_{0}(\tau)=A_{0}^{0}\left(\frac{D_{0}}{D(\tau)}\right)^{1 / 2}, \\
\psi^{(1)}(\tau)=\frac{1}{2} \int_{0}^{\tau} \imath \operatorname{Im}(\epsilon(\mathbf{r}(\tilde{\tau}))) \mathrm{d} \tilde{\tau}
\end{array}
$$

where $A_{0}$ and $\psi^{(1)}$ are the ray amplitude and ray phase terms, respectively, $A_{0}^{0}$ is an initial amplitude, $D$ denotes the determinant of the Jacobian of the coordinate transform from the ray phase space to the configuration space, $\tau$ is the arc-length ray parameter, $\psi^{(1)}$ is the ray phase perturbation term and $\mathbf{r}$ denotes the ray coordinate. Note here that the equation for the ray field would differ for $\mathrm{CGO}$, in which case $\tau, A_{0}$ and $\mathbf{r}$ may be complex-valued and $\psi^{(1)}$ is no longer a perturbation term.

The ray field diverges at caustics and in a region of space around caustics such that the phase difference $\Delta \psi$ between two rays reaching it is less than $\lambda_{0} / 3$. In these regions, IFRIIT implements an EI technique to compute caustic fields from the ray phase and ray amplitude terms, despite the latter being diverging. The EI method models the caustic field by a sum of an Airy function and the derivative of an Airy function. This approximation is valid for most Fold-type caustics, even in presence of perturbations such as curvature and departure from a linear permittivity profile.

\section{PRINCIPLES OF COMBINING AMR AND IRT IN IFRIIT}

In the IFRIIT framework, all quantities are described using a piece-wise linear approximation on a 3-D tetra- 
hedral mesh and gradients are computed from nodal values. This approximation is employed to ensure numerical efficiency with respect to inline modeling. In relation to the IRT algorithms, the vertices of the tetrahedra are observation points, e.g. spatial locations where the mapping from ray phase space to configuration space is computed. While hydrodynamic quantities are restricted to the piece-wise linear approximation, since it is prescribed by the external input, laser-related quantities (fields, wavevectors, permittivity perturbations) can be refined beyond the piece-wise linear approximation. This is mainly for two reasons: (i) laser-related quantities do not linearly depend on ray phase space parameters (especially at caustics), and (ii) ray phase space parameters may not vary linearly at subgrid scales, because they depend on cumulative effects along whole ray trajectories. While a discrepancy between subgrid fields and piecewise linear fields is not an issue for collisional absorption computations, it can play a role for CBET or hot electron generation.

Here, we take advantage of the IRT framework to add new observation points in the mesh, until there is an effective convergence between the piece-wise linear scheme at large scales and the non-discretized underlying fields. Typically, a laser caustic may occur at any point in the plasma independently of the initial meshing applied. This sharp boundary is rarely captured by the mesh. While a full mesh resolution scaling could allow to numerically describe this caustic, it is much more efficient to only refine the region of interest. The iterative AMR scheme implemented in IFRIIT is specific in that it works directly on tetrahedrons, does not modify laser refraction during refinement, and does not introduce preferential directions for subgrid-gradients. Details about the implementation are given in App. A.

\section{CBET IN THE GEOMETRICAL OPTICS DESCRIPTION OF LASER FIELDS}

The laser propagation model summarized in Sec. III gives access to the electric field distribution in the plasma. Its coupling with the AMR framework presented in Sec. IV and App. A allows adaptive mesh refinement of gradients, e.g. in laser fields. We now formulate a model describing the CBET occurring between overlapped laser waves in a plasma.

\section{A. Form of the dielectric permittivity function}

CBET manifest itself from a low frequency density perturbation that parametrically grows to large amplitudes. Depending on the plasma response, these perturbations $\delta n$ may (i) retard or accelerate the laser wave phasefront and (ii) amplify or attenuate the amplitude. These effects occur for the field components which are aligned with the pump polarization vector, such that the pump- beam/plasma system can rotate the probe beam polarization vector ${ }^{26}$. For simplicity, we consider in this paper wavefields interacting with aligned polarization vectors. A simple extension to distributed polarization rotation can be obtained by following the formalism described in Ref. 27. More accurate description of polarizations in IFRIIT, including their rotation through CBET, will be covered in future work.

Substituting $n_{e}$ by $n_{e}+\delta n$, the dielectric permittivity seen by wavefield $m$ overlapping wavefield $n$ reads:

$$
\begin{gathered}
\operatorname{Re}(\epsilon)=1-\frac{n_{e} / n_{c}}{1+\left(\nu_{\mathrm{IB}} / \omega\right)^{2}}\left(1+\frac{\operatorname{Re}(\delta n)}{n_{e}}\right), \\
\operatorname{Im}(\epsilon)=\frac{n_{e} / n_{c}}{1+\left(\nu_{\mathrm{IB}} / \omega\right)^{2}}\left(\frac{\nu_{\mathrm{IB}}}{\omega}-\frac{\operatorname{Im}(\delta n)}{n_{e}}\right),
\end{gathered}
$$

where we have neglected cross terms involving $\nu_{\mathrm{IB}} \delta n$. It is readily seen that the IAW perturbation affects both the real and imaginary components of the wavefield phase, inducing potential effects of phase delay/acceleration, and field amplitude attenuation/gain.

There are several ways to resolve these equations in the IFRIIT framework. The most accurate resolution of the ray equations would involve CGO, thus modeling wavefield refraction on both the real and imaginary part of the density perturbation, in addition to wavefield attenuation or gain. In the GO framework, $\operatorname{Im}(\epsilon)$ is a perturbative term and does not affect the ray trajectory. The latter is only affected by $\operatorname{Re}(\delta n)$, which implies that trajectory and $\delta n$ calculations must be conducted iteratively. Here, we make the additional simplification that $\operatorname{Re}(\delta n) / n_{e} \ll 1$. This allows a dramatic reduction in numerical costs for IRT since only the phase perturbation integral needs to be conducted iteratively (Eq. (5)) for pump depletion computations. This simplification is also consistent with the AMR framework proposed in this paper.

\section{B. Multi-sheeted covered spaces}

The formulation presented in V A relates to two wavefields overlapping. The extension of this formulation to one wavefield overlapping with many other wavefields is straightforward in the limit where we have assumed the dielectric permittivity perturbation to be isotropic (i.e. we neglect polarization rotation). The total density perturbation seen by a wavefield is the sum of each pair of perturbations with the other overlapping wavefields. It is crucial here to understand how the summation must be performed, considering that a beam may in theory interact with itself through this process.

In the ray-optics framework, a mapping is built between the ray coordinate phase space and the configuration space. This mapping may be subdivided in phase space between many bijective sub-mappings called ray sheets, denoted as $\Omega$. It is useful here to give an example: consider a plane wave incident on a linear density 
gradient; in absence of strong non-linearity in the density response, the total field in the medium is the overlap of a forward and a backward propagating EM wave. The set of ray parameters corresponding to the forward wave constitutes the first ray sheet, while those of the backward wave are in the second ray sheet. Note that there may be more than 2 ray sheets in more generic configurations.

In order to accurately describe non-linear effects involving coupling between wavefields, one must in theory account for the interaction between fields described on different sheets, even within the same beam. As such, the density perturbation $[\delta n]_{\Omega_{s}^{b}}$ seen by a ray from beam $b$, propagating within the phase space parameters of sheet $s$, denoted $\Omega_{s}^{b}$, is:

$$
[\delta n]_{\Omega_{s}^{b}}=\sum_{\substack{\hat{b}=1 \\ \hat{b} \neq b}}^{N_{B}} \sum_{\hat{s}=1}^{N_{S}^{\hat{b}}}[\delta n]_{\Omega_{s}^{b} \Omega_{\hat{s}}^{\hat{b}}}+\sum_{\substack{\hat{s}=1 \\ \hat{s} \neq s}}^{N_{S}^{b}}[\delta n]_{\Omega_{s}^{b} \Omega_{\hat{s}}^{b}}
$$

where $N_{B}$ is the number of beams, $N_{S}^{b}$ is the number of sheets in beam $b$, and $[\delta n]_{\Omega_{s}^{b} \Omega_{\hat{s}}^{\hat{b}}}$ is the density perturbation originating from the overlap of sheet $s$ of beam $b$ with sheet $\hat{s}$ of beam $\hat{b}$. The preferred formulation of $\delta n$ used in IFRIIT is a linear kinetic model, that is summarized in App. B 1. A fluid model has also been implemented, see App. B 2.

Computation of the ray phase, which requires integration of $\operatorname{Im}(\epsilon)$ along ray trajectories, is conducted on each sheet separately. When reaching a caustic, the ray changes sheets and the permittivity it sees is changed following Eq. (8). This requires tracking the coordinates of the caustic surface in $3-D$ phase space. In typical ICF situations, one should expect presence of 1 or 2 caustics per beam at most.

\section{Multi-sheeted Cross-Beam Energy Transfer}

We now give an illustration of CBET in a simple multisheet case. Consider the case given in Fig. 1 (a), of a laser beam propagating at normal incidence in a permittivity profile of initial form $\epsilon=1-n_{e} / n_{c} /\left(1+\imath \nu_{\mathrm{IB}} / \omega_{0}\right)$, where $n_{e} / n_{c}=x / L$ and $\nu_{\mathrm{IB}}=n_{\mathrm{e}} / n_{\mathrm{c}} \nu_{\mathrm{IB}}^{*}$ with $\nu_{\mathrm{IB}}^{*}$ the Inverse Bremsstrahlung (IB) frequency at the critical density. The field solution in the density profile is a superposition of an incident and reflected wavefield. A linear flow velocity profile of the form $V(x)=V_{0}-x / L_{V}$ is added, with $V_{0}=1.1 C_{s}, L_{V}$ a velocity scalelength, and $C_{s}=\sqrt{k_{\mathrm{B}}\left(\langle Z\rangle T_{e}+3 T_{i}\right) / M}$ the sound speed, with $M$ the ion mass, $T_{e}$ and $T_{i}$ the electron and ion temperatures, respectively, $\langle Z\rangle$ the average ionization rate and $k_{\mathrm{B}}$ the Boltzmann constant. In the plasma reference frame, the incident and reflected field frequencies are up- and down-shifted, respectively, such that the incident wavefield transfers energy to the reflected wavefield. We consider two cases: (i) a steep density profile case with

\begin{tabular}{|l||c|c|c|}
\hline Short-scalelength case & $\left|u_{\text {ref }}\right|$ & $\left|u_{\text {cau }}\right|$ & $P_{\text {abs }}(\%)$ \\
\hline LPSE & $5.12 \times 10^{-3}$ & $1.04 \times 10^{-2}$ & 41.8 \\
IFRIIT, self CBET & $5.09 \times 10^{-3}$ & $1.06 \times 10^{-2}$ & 42.5 \\
IFRIIT, no CBET & $2.30 \times 10^{-3}$ & $2.12 \times 10^{-2}$ & 88.3 \\
\hline \hline Long-scalelength case & $\left|u_{\text {ref }}\right|$ & $\left|u_{\text {cau }}\right|$ & $P_{\text {abs }}(\%)$ \\
\hline LPSE & $2.43 \times 10^{-3}$ & $1.23 \times 10^{-3}$ & 87.7 \\
IFRIIT, self CBET & $2.35 \times 10^{-3}$ & $1.26 \times 10^{-3}$ & 86.9 \\
IFRIIT, no CBET & 0 & $1.38 \times 10^{-3}$ & 100 \\
\hline
\end{tabular}

TABLE I. Summary of simulation results for the shortscalelength and long-scalelength cases. Results are given for the reflected field amplitude $\left|u_{\text {ref }}\right|$, peak caustic field amplitude $\left|u_{\text {cau }}\right|$ and power absorption fraction $P_{\text {abs }}$, for calculations using LPSE and IFRIIT with and without self-CBET.

$L=30 \mu \mathrm{m}, L_{V}=80 \mu \mathrm{m}$ and $\nu_{\mathrm{IB}}^{*}=10 / \mathrm{ps}$, relevant to earlytime interaction (e.g. the picket pulse employed in ICF), and (ii) an average scalelength case with $L=300 \mu \mathrm{m}$, $L_{V}=800 \mu \mathrm{m}$, and $\nu_{\mathrm{IB}}^{*}=7 / \mathrm{ps}$. Results from IFRIIT are compared to the wave solver $L P S E^{22}$. For consistency with respect to the LPSE implementation, we use the fluid IAW response in IFRIIT (see App. B 2). The IAW damping rate is fixed in both codes to $\nu_{s}=\omega_{s} / 10$, with $\omega_{s}$ the frequency of the IAW. In both cases, the incident laser intensity is $5 \times 10^{14} \mathrm{~W} / \mathrm{cm}^{2}$ at $3 \omega$.

The steady-state solution in IFRIIT is obtained by successive iteration of the phase integration step (Eq. 5) until stabilization of the field solution. For this numerically challenging configuration, where the wavefield after reflection extracts significant energy from the incident wavefield itself, the iterative algorithm may oscillate between two periodic solutions instead of converging. As such, we have found necessary to implement numerical damping in the iterative process. The permittivity perturbation at iterative step $n+1$ and used in the IFRIIT calculation, noted $\delta \epsilon^{(n+1)}$, is computed as $\delta \epsilon^{(n+1)}=\delta \epsilon^{(n)}+0.25\left(\Delta \epsilon-\delta \epsilon^{(n)}\right)$, where $\Delta \epsilon$ is the instantaneous permittivity perturbation computed at step $n$. Simply, only $25 \%$ of the calculated perturbation is updated into the field calculation at each iterative step.

Fields calculated with LPSE and IFRIIT are shown in Figs. $2(\mathrm{a}, \mathrm{b})$. The LPSE field solution in the shortscalelength case (Fig. 2 (a)) is oscillating in time, such that the caustic field varies to within $10 \%$. Here, IFRIIT produces a steady-state solution that is seen to be contained within the bounds of the LPSE temporal oscillation. In both scalelength cases, IFRIIT reproduces the laser fields to within \%-level error, as summarized in Tab. I. Most notably, the scattered fields and caustic fields are in excellent agreement. Results obtained with IFRIIT when neglecting the self-CBET interaction are given in Figs. 2 (c,d). In absence of self-CBET modeling, the scattered field may be entirely absorbed in plasma, thus leading to high absorption fractions. Accounting for selfCBET increases the reflectivity of the plasma by $40 \%$ in the short scalelength case and $13 \%$ in the long scalelength case. In the former, the caustic fields are also seen to be twice lower in the presence of CBET, an effect that is 
(a)

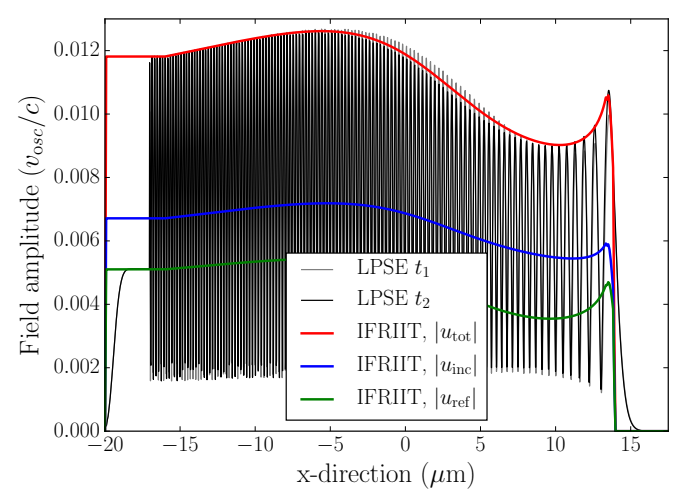

(c)

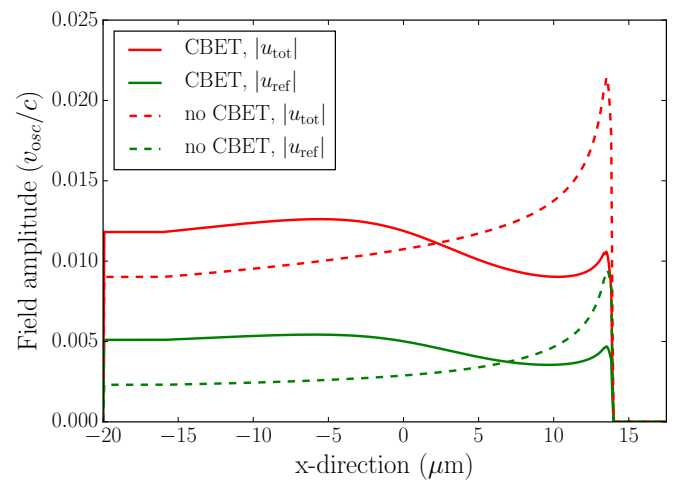

(b)

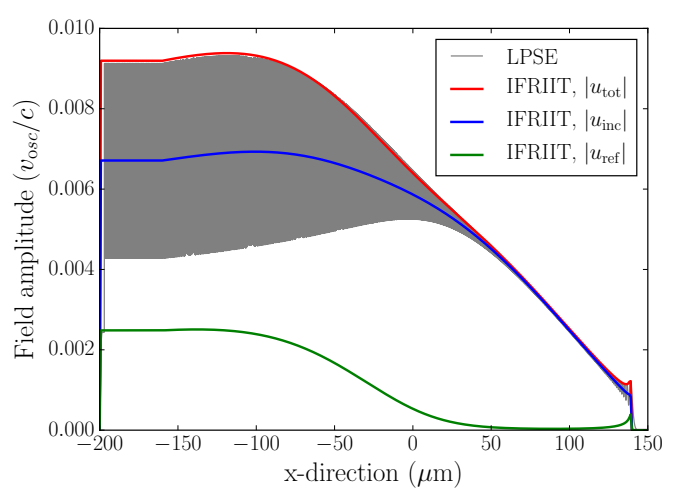

(d)

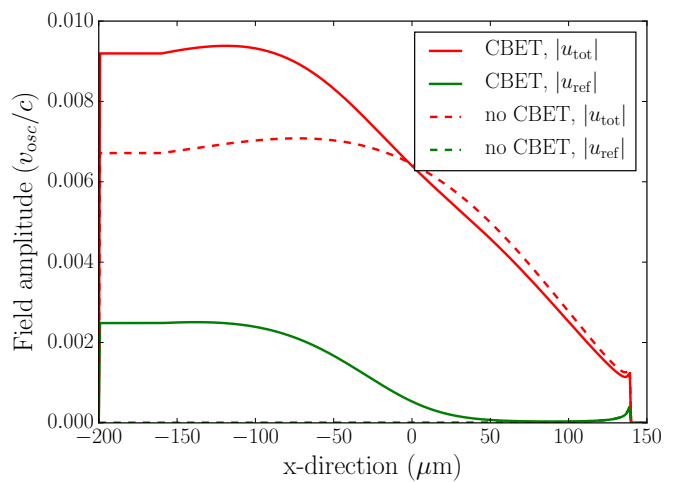

FIG. 2. Comparison of fields from LPSE and IFRIIT for the (a) short-scalelength and (b) long-scalelength cases. LPSE fields in the short-scalelength case (a) are taken at two times $t_{1}$ and $t_{2}$ such that the caustic field reaches a maximum and a minimum. These are shown in grey and black, respectively. LPSE fields in the long-scalelength case (b) do not exhibit temporal oscillation and are shown as a grey solid line. (c,d) Comparison of fields from IFRIIT with (solid lines) and without (dashed lines) self-CBET accounted for, in the short-scalelength and long-scalelength cases, respectively. In all figures, fields from IFRIIT are shown in colors: incident fields $\left|u_{\text {inc }}\right|$ in blue, reflected fields $\left|u_{\text {reff }}\right|$ in green and total fields $\left|u_{\text {tot }}\right|=\left|u_{\text {inc }}\right|+\left|u_{\text {ref }}\right|$ in red.

important to account for when computing other LPIs.

\section{ADAPTIVE MESH REFINEMENT APPLIED TO CBET MODELING IN 3-D}

\section{A. Motivation}

Laser light propagating in plasmas often involves sharp features in laser-related quantities, such as fields at caustics, narrow resonance profiles for LPIs or discrete overlaps of wavefields. These gradients are often sub-grid scale to hydrodynamic cells, positioned at arbitrary locations, and rarely fall along mesh points. Applying traditional inline CBET models relying on rigid-scale estimators to such configurations often leads to spurious effects, such as oscillations of gains along the beam profiles and difficulties in conserving energy. This is notably what prompted the formulation of the recent Caustic Gain Truncation (CGT) method ${ }^{28}$. Here we show that AMR can be used to control both the energy conservation and the local gain convergence.

\section{B. CBET convergence with IRT and AMR}

Consider the case given in Fig. 1 (b) of a 1-D gain configuration, i.e. the interaction between two 1-D waves (without transverse profile) overlapping over a fixed distance $L$, reproduced in a 3-D geometry. In order for the latter to be equivalent to 1-D, several geometrical requirements must be met: (i) beam profiles must be infinitely sharp top-hats of width $L \sin (\theta / 2)$ (with $\theta$ the crossing angle) and the beam axes must be crossing within the same plane; (ii) the plasma profile seen by each beam along its propagation axis must be identical; and (iii) the beam interaction volume must be entirely inside the simulation box. In that configuration, the probe gain rate inside the interaction volume is constant, and each ray in each beam sees the same amplification length $L$. The spatial gain along the beam profile is then also a top-hat, following the initial beam profile.

The challenge in reproducing a 1-D gain profile from a 3-D interaction is that, in the general case, the convex hull of the interaction volume is not aligned with mesh grid-points. If the number of cells in the homogeneous region is not much higher than the number of 
cells crossing the interaction volume boundary, the local gains will be erroneous and energy conservation will not be obtained. In the ideal situation, cell surfaces in 3-D should be aligned with the sharp boundary.

We consider a non-dissipative $\mathrm{C}_{5} \mathrm{H}_{12}$ plasma at $n_{e} / n_{c}=0.15, T_{e}=1.8 \mathrm{keV}, T_{i}=0.3 \mathrm{keV}$, and two beams crossing at an angle of $\theta=25^{\circ}$ with square flat-top profile of $650 \mu \mathrm{m}$ radius. The beams are detuned in the $[0,3] \AA$ range. The pump intensity is $I_{\text {pump }}=6.7 \times 10^{13} \mathrm{~W} / \mathrm{cm}^{2}$ and the probe intensity is set to $I_{\text {probe }}=10^{-10} I_{\text {pump }}=$ $6.7 \times 10^{3} \mathrm{~W} / \mathrm{cm}^{2}$, so that no significant pump depletion occurs below gains of 20 . The simulation domain is a $9000 \times 5000 \times 5000 \mu \mathrm{m}$ box of $15^{3}$ mesh points. In this configuration, there are approximately equal number of cells initially inside the interaction volume and cells that encompass the interaction volume interface. In order to avoid favoring the AMR algorithm geometrically, the beams are purposefully misaligned with the $y$ mesh direction such that $\mathbf{k}_{0} \cdot \mathbf{e}_{y}=\cos \left(20^{\circ}\right)$. The refinement criterion (Eq. A1) is applied on the imaginary component of the permittivity perturbation, per sheet, $\operatorname{Im}\left([\delta \epsilon]_{\mathrm{CBET}}\right)=\operatorname{Im}\left([\delta n]_{\Omega_{s}^{b}}\right) / n_{c} /\left(1+\left(\nu_{\mathrm{IB}} / \omega_{0}\right)^{2}\right)$. The IAW response is described with the linear kinetic model (see App. B 1).

The CBET gain integrated on the transverse beam profile as a function of adaptive mesh refinement depth $m$ and beam detuning is shown in Fig. 3 (a). The integrated gain converges toward the 1-D linear gain with the refinement depth $m$. Scalings of energy conservation error are shown in Figs. 3 (b-c), including values obtained at lower and higher gains (by changing the pump beam intensity). Increasing the refinement depth allows one to decrease the energy conservation error. Both the energy conservation and gain trends are related to how the interaction volume is gradually regularized: the interaction length seen by each ray in each beam statistically converges toward the $1-\mathrm{D}$ value through iterative splitting of tetrahedra along the boundary.

Local gains across the 2-D transverse beam profile are shown in Fig. 4. Without mesh refinement (Fig. 4 (a)), the spatial gain is purely determined by the geometrical arrangement of the beams with respect to the mesh vertices. While the integrated gain is only $10 \%$ below the reference, the local gain varies from $-30 \%$ below to $+10 \%$ with large spatial variations. This may be detrimental to 3 -D calculations where illumination symmetry is studied, as these variations will imprint unphysical low mode perturbations. Increasing the refinement depth gradually smooths transverse gain variations in the beam profile (Figs. 4 (b-d)). While gain deviations are still present on the beams edges, these are related to the infinitely steep laser profiles (top-hat beams) and would be smoothed out in simulations with realistic exponential-like profiles. An illustration of the mesh refinement is given in Fig. 5 , showing a cut in the $y=0$ plane of the probe beam field amplitude for $m=0$ and $m=3$. In this particular test, it is found that an energy conservation error mostly below $1 \%$ of the exchanged energy can be obtained up to gains of 20, with a refinement depth of $m=4$.

In general, we have observed the following convergence trends with the refinement depth: (i) gains converge linearly, (ii) energy conservation error converges quadratically and (iii) standard deviation of the local gain transverse to the beam profile converges sub-linearly. These results illustrate how adaptive meshing used in conjunction with IRT is a promising approach for accurate gain modeling on local and global scales, as well as controlling energy conservation without usage of non-physical numerical parameters.

\section{DIRECT-DRIVE LIKE CBET CALCULATIONS WITH IFRIIT}

We now consider CBET calculations in the presence of high refraction rates and caustics. The plasma density is set to a 1/4 OMEGA scale ICF profile in 2-D:

$$
\frac{n_{\mathrm{e}}}{n_{\mathrm{c}}}=\frac{n_{\mathrm{e}, \max }}{n_{\mathrm{c}}} \exp \left[-\frac{\left(\mathbf{r}-\mathbf{r}_{0}\right)^{2}}{L_{n}^{2}}\right]
$$

with $L_{n}=83.6 \mu \mathrm{m}, n_{\mathrm{e}, \max }=4 n_{c}$ and $\mathbf{r}_{0}=(25,25,0)$ $\mu \mathrm{m}$. The reduced scale was chosen for efficiency of the reference calculation with $L P S E$.

For consistency with full OMEGA-scale implosions, the IB frequency at the critical density is set to the large value of $\nu_{\mathrm{IB}}^{*}=40 / \mathrm{ps}$. The density profile is Gaussian in the $(x, y)$ plane and invariant along the $z$ direction, to facilitate comparisons with LPSE. The IAW are described using a fluid response with prescribed amplitude damping rate of $\nu_{s}=\omega_{s} / 10$ (see App. B 2). The plasma flow follows a Gaussian profile:

$$
\mathbf{u}(\mathbf{r})=\frac{\mathbf{r}-\mathbf{r}_{0}}{\left\|\mathbf{r}-\mathbf{r}_{0}\right\|}\left(V_{\max }\left(1-\exp \left[-\frac{\left(\mathbf{r}-\mathbf{r}_{0}\right)^{2}}{L_{v}^{2}}\right]\right)+V_{\min }\right)
$$

with $L_{v}=170.2 \mu \mathrm{m}, V_{\max }=2.5 C_{s}$ and $V_{\min }=0.01 C_{s}$.

Two comparisons are conducted; two-wave mixing where one beam at $3 \times 10^{15} \mathrm{~W} / \mathrm{cm}^{2}$ interacts with itself near the caustic and four-wave mixing where two $2 \times 10^{15}$ $\mathrm{W} / \mathrm{cm}^{2}$ beams cross at $90^{\circ}$. In all cases, the beams are super-Gaussian order 4 with radius at 1/e of $100.9 \mu \mathrm{m}$ at the wavelength of $351 \mathrm{~nm}$.

\section{A. Single-beam self-CBET in exponential density gradient}

We first consider the case given in Fig. 1 (c) of one beam propagating in the cylindrical density profile. As in Sec. VC, the beam interacts with itself through the multiple sheets describing the ray manifold. A comparison of fields between LPSE and IFRIIT in the whole domain is shown in Fig. 6 (a), where an excellent overall agreement is found. A line-out taken at the boundary of the simulation domain is shown in Fig. 6 (b). Separation of the incident and reflected field in IFRIIT shows 
(a)

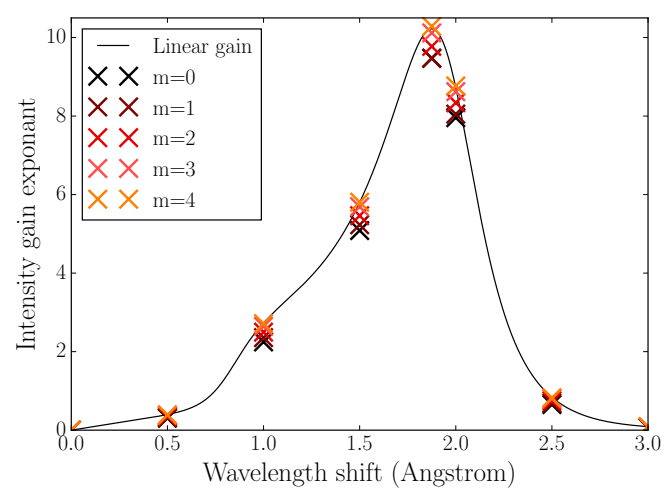

(b)

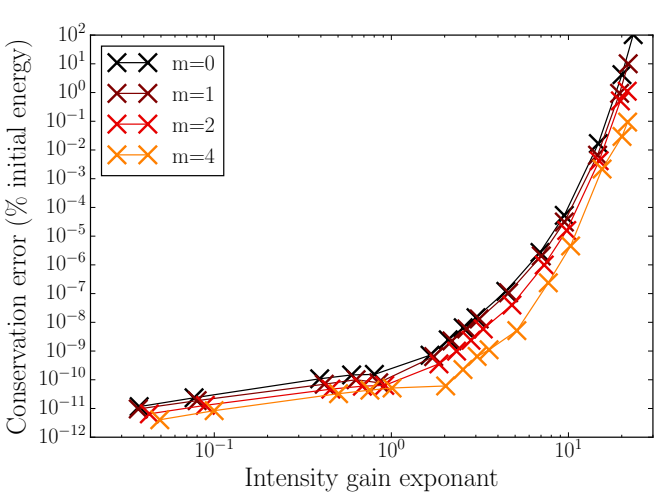

(c)

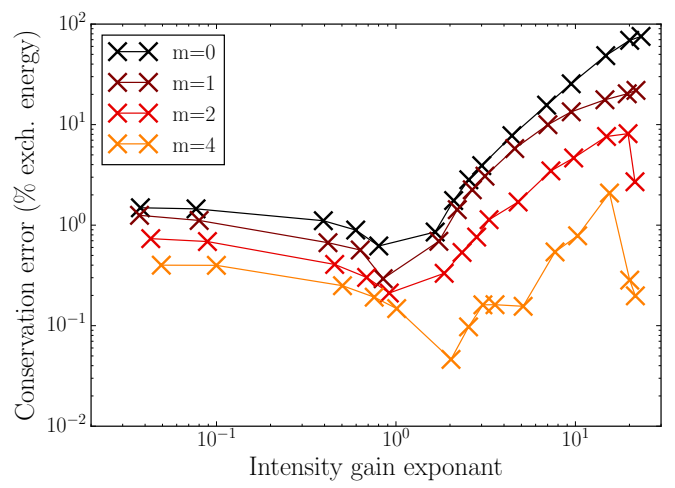

FIG. 3. (a) Linear gain as a function of wavelength detuning between the beams. The reference 1-D gain is shown as a black line. Gains obtained with IFRIIT using the unrefined mesh are shown as black crosses. The scaling from refinement depth $m=1$ to $m=4$ is shown as colored crosses. (b-c) Energy conservation error of the CBET interaction as a function of gain, (b) in \% of the initial energy and (c) in \% of the exchanged energy. Results from the unrefined mesh configuration are shown in black. Scaling from $m=1$ to $m=4$ are shown as colored lines.

that the light is scattered almost uniformly along $\sim 1.3 \pi$ radians, totaling a scattered power of $12.7 \%$. The same computation without CBET yields $3.9 \%$ scattered power, meaning that almost all the laser energy is absorbed in the plasma.

A profile of the caustic field along the $0.36 n_{c}$ contour is given in Fig. 6 (c). IFRIIT correctly reproduces the caustic field predicted by the wave solver, in amplitude and position, even at rather low mesh resolutions. While the Etalon Integral ${ }^{29}$ implemented in IFRIIT was modified and validated for both non-dissipative and dissipative media (see Ref. 21), these results show that the technique remains valid when in presence of both dissipation and amplification. Comparing fields obtained with and without CBET underlines how self-CBET within a beam is key to describing the field amplitude correctly: self-CBET depletes the caustic field by a factor of 2 and increases the on-axis field by about $15 \%$. Good agreement is also found for the backscattered fields, as shown in Fig. 6 (d).

The side-scattered fields, obtained far from the beam axis, are shown in Figs. $6(\mathrm{e}, \mathrm{f})$. Although IFRIIT exhibits good overall agreement with $L P S E$, large and small wavelength perturbations are present. These side- scattered fields are formed after significant propagation in the caustic region, where the incident field transfers energy to the reflected field (see Figs. 7 (a,b)). The largewavelength perturbation, i.e. discrepancy in location and amplitude of minimum and maximum, is thought to be due in part to the difficulty to accurately model such high rates of refraction in LPSE. Any small error is amplified through CBET and can lead to a spatial shift. Another factor that may play a role is the fact that IAW advection is neglected in IFRIIT (i.e. they are assumed to be stationary), while this is not the case in LPSE. The small wavelength perturbations are caused by the piecewise linear scheme in IFRIIT, which introduces noise amplified by CBET depending on the configuration of the tetrahedra at the caustic. The latter can be improved through AMR, as shown in Fig. 6 (f). The corresponding adaptive meshing of the caustic region is shown in Fig. 7 (c).

Energy conservation in this case is mainly affected by how well the caustic region, which truncates self-CBET for the side-scatter, is resolved by the mesh and the piecewise linear approximation. Notably, when the caustic is under-resolved, rays may propagate in tetrahedra with ill-computed gradients from vertices far in the caustic 
(a)

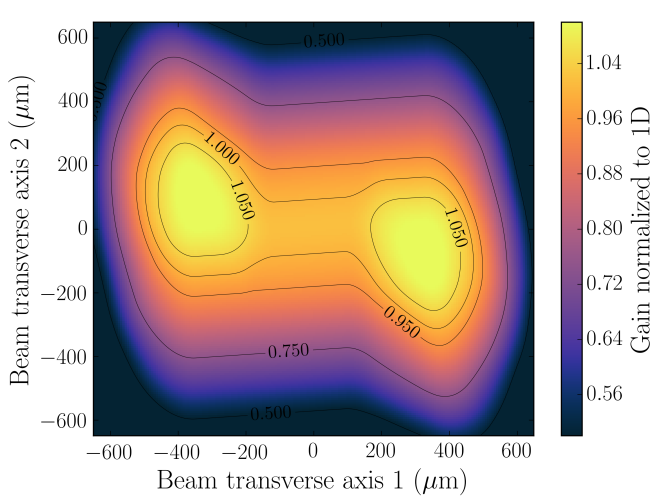

(c)

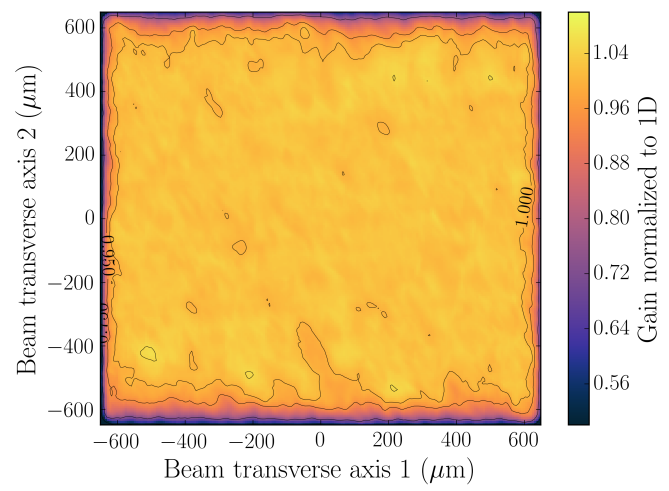

(b)

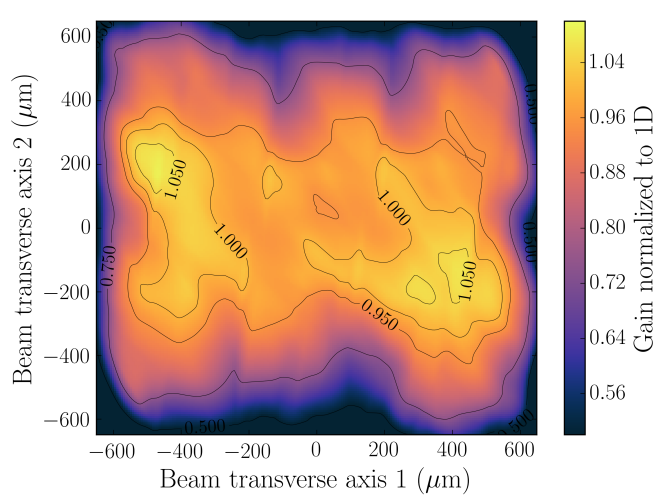

(d)

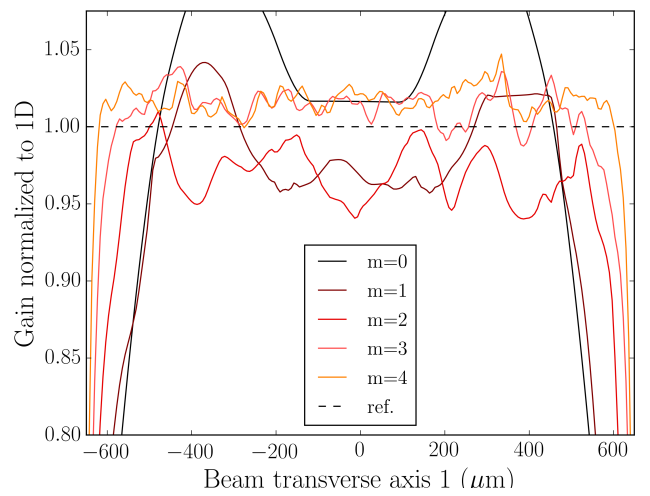

FIG. 4. (a-c) Intensity gain exponent along the probe beam transverse directions after the interaction. The refinement depth is (a) $m=0$; (b) $m=1$ and (c) $m=4$. Isocontours are shown at 75, 95, 100 and $105 \%$. (d) Profiles of intensity gain exponent along one transverse direction of the probe beam for $m=0$ to $m=4$. The reference solution is shown as a black dashed line. For all plots, gains are normalized to the reference 1-D gain.

(a)

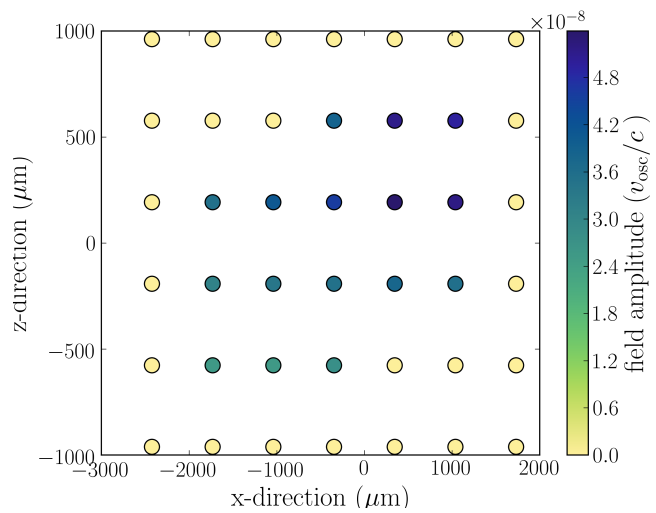

(b)

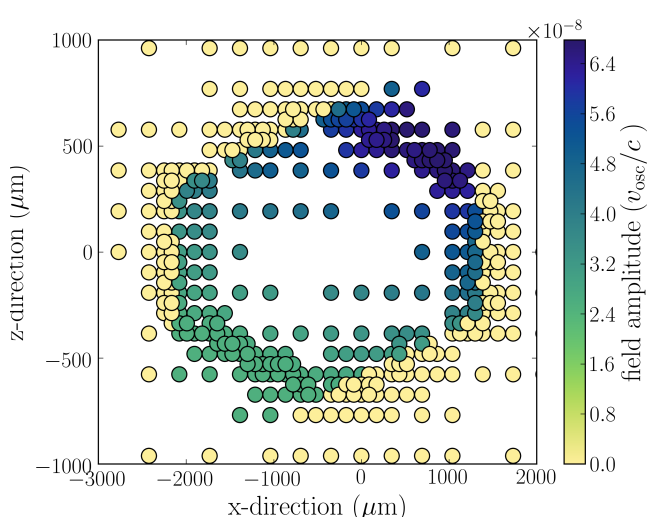

FIG. 5. Probe beam field amplitude in the (x-z) plane, for (a) the unrefined initial mesh and (b) the mesh after 3 iterations of the refinement algorithm. Each dot represents the vertex of a tetrahedron and is an observation point for the IRT algorithm.

shadow. Increasing mesh resolution or AMR depth are two strategies that naturally improve energy conservation in that context. While increasing the global mesh resolution is only relevant if the input hydrodynamic data itself is provided on a finer grid, increasing AMR depth is always possible. A scaling of energy conservation error versus total CPU time as a function of global mesh resolution scaling and AMR depth scaling is given in Fig. 7 (d). It is found that AMR provides better convergence rates compared to a full scaling. Notably, reaching energy conservation of $0.2 \%$ of the exchanged energy is done in at most two refinement steps even at modest resolutions. It is important to note here that the AMR is refining the mesh in the $3-\mathrm{D}$ volume ${ }^{32}$, whereas the mesh resolution scaling is conducted only in the $(x, y) 2$-D plane. As such, the numbers given here artificially favor the full 
(a)

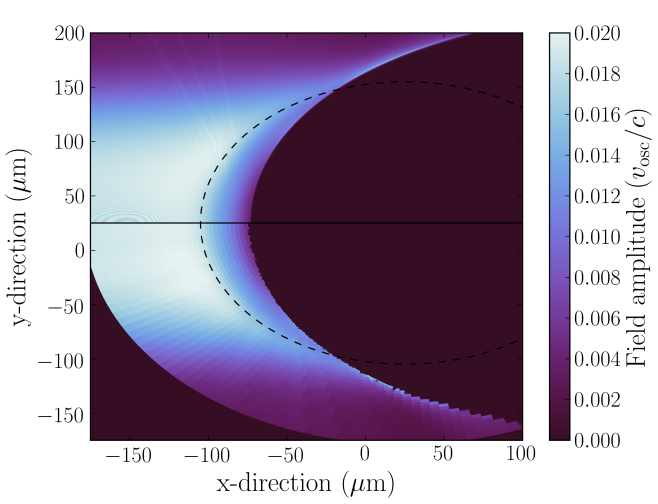

(c)

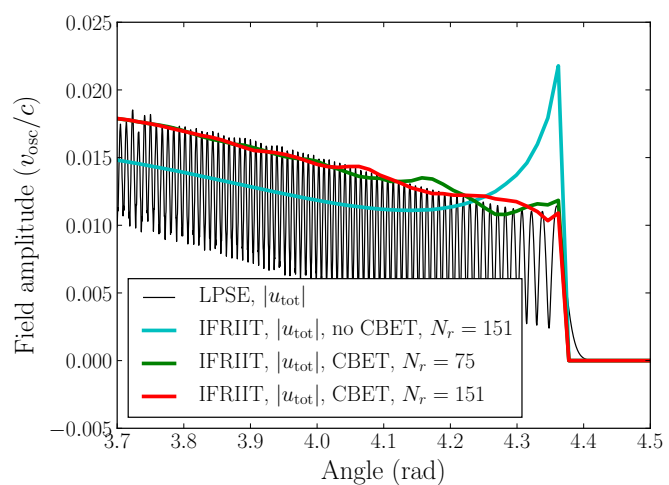

(e)

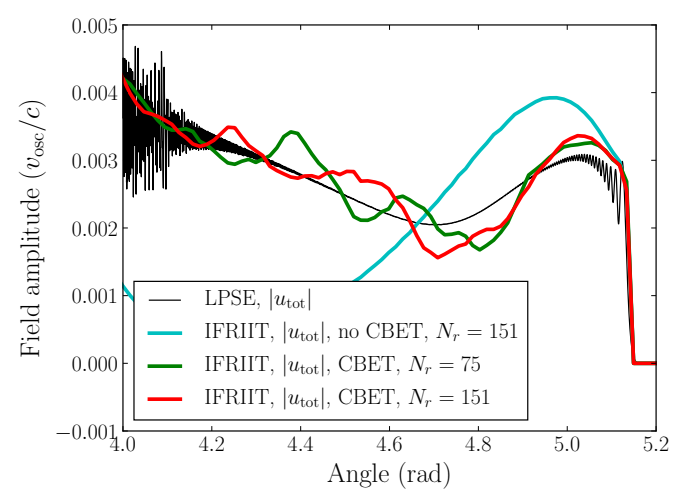

(b)

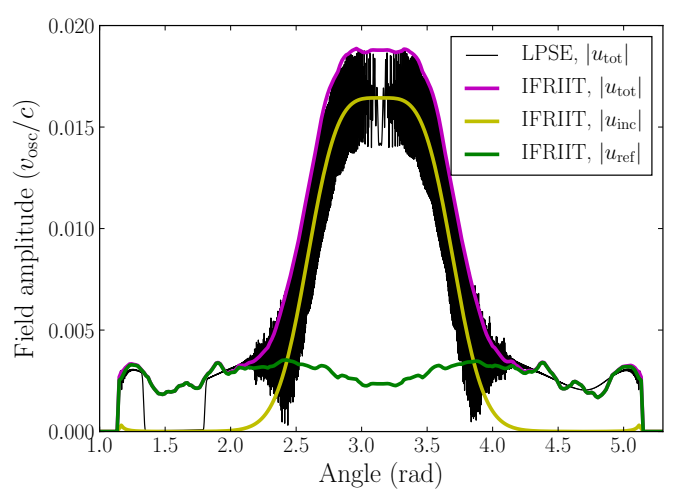

(d)

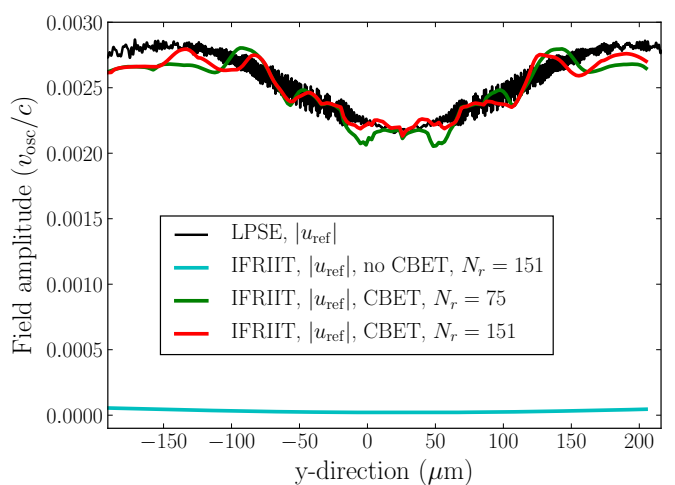

(f)

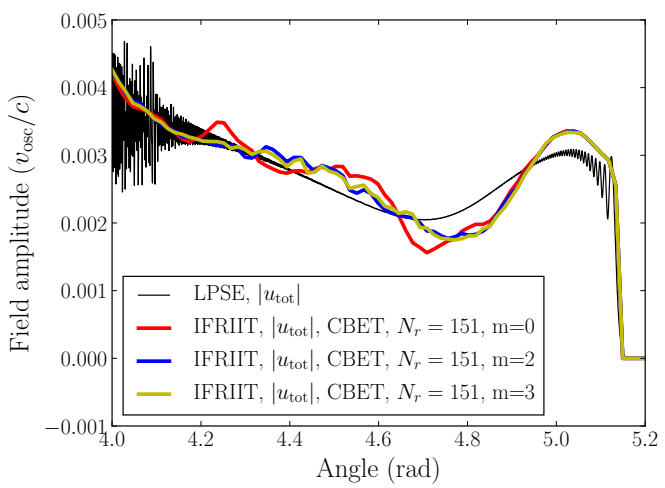

FIG. 6. (a) Total laser field in the single-beam 2D Gaussian density case. Fields from LPSE and IFRIIT are above and below the black line at $y=25 \mu \mathrm{m}$, respectively. For this panel, fields below $0.001 n_{c}$ were not computed in IFRIIT, hence the asymmetry. Mesh resolution is 14 cells per wavelength for $\operatorname{LPSE}\left(18000^{2}\right)$ and $N_{r} \times N_{\theta} \times N_{z}=75 \times 181 \times 5$. The dashed circle indicates the position of the $0.36 n_{c}$ isocontour. (b) Total laser field encircling the simulation domain at radius of $200 \mu \mathrm{m}$, for LPSE in black and IFRIIT in magenta. Decompositions in incident and reflected fields are shown in yellow and green, respectively, for IFRIIT . (c) Close up of the caustic fields along the $0.36 n_{c}$ isocontour as a function of an angular coordinate, with $\pi$ corresponding to the input beam direction. A simulation without CBET is shown as a cyan line. Simulations with $N_{r}=75$ and $N_{r}=151$ are shown in green and red, respectively. No AMR is used. (d) Back-scattered and (e) side-scattered fields. Figure legend is the same as in (b). (f) Side scattered fields scaling with AMR depth $m$, for $N_{r}=151$ and $m=0$ (red), $m=2$ (blue) and $m=3$ (yellow).

mesh scaling.

At fixed initial mesh resolution, the AMR step provides control over energy conservation. Because this method works by refining the finite-element grid for the whole IFRIIT algorithm, there is no need to introduce artificial correction factors. Notably, there is no need to renor- malize laser energy to enforce energy conservation, as is sometime done in CBET codes. In the case presented here, most of the refinement is occurring at the caustic. This is consistent with conclusions presented in Ref. 28, where Caustic Gain Truncation (CGT) was proposed to increase convergence rates by tracking the caustic loca- 
(a)

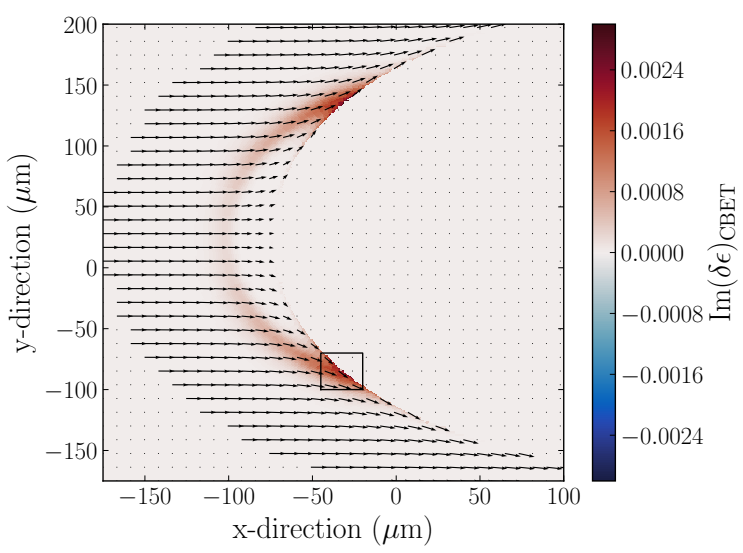

(c)

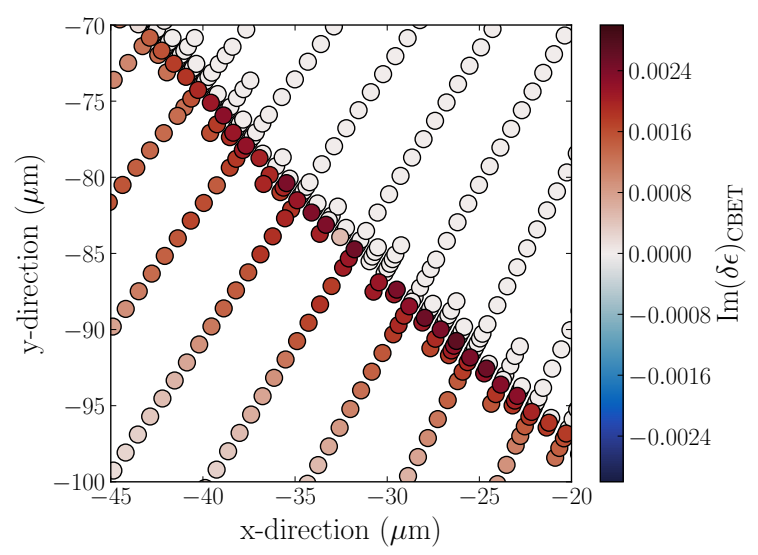

(b)

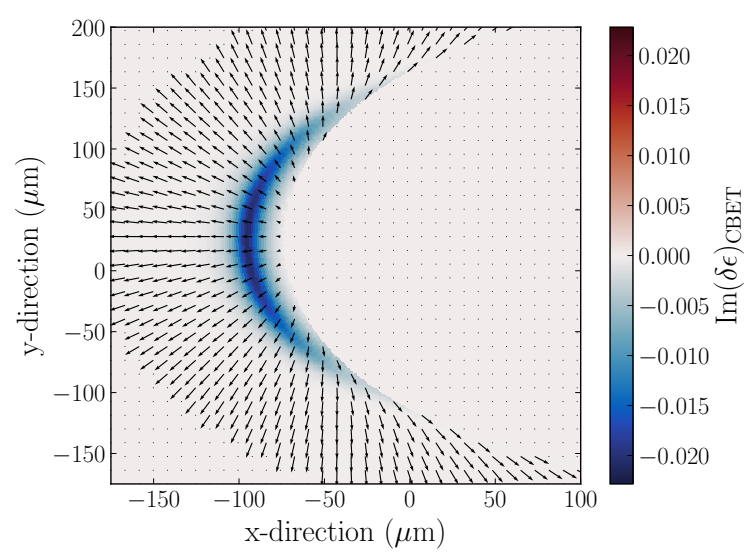

(d)

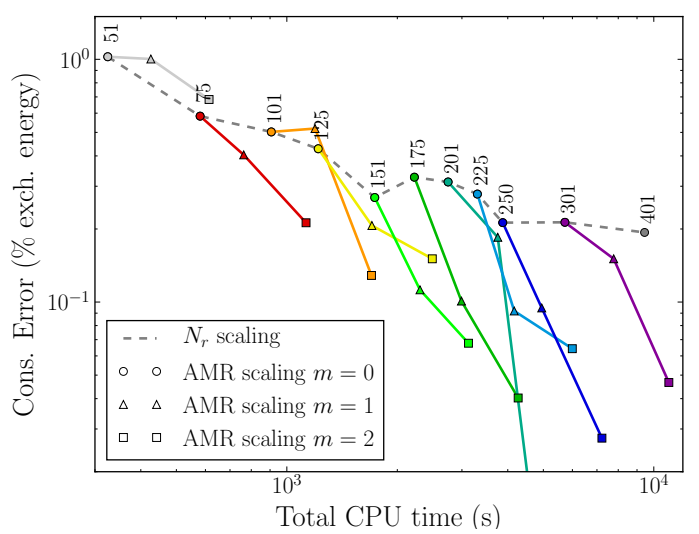

FIG. 7. Imaginary part of the permittivity perturbation for the (a) first sheet (incident field) and (b) second sheet (reflected field), as computed by IFRIIT. Positive values correspond to depletion, and negative values to amplification. The k-vectors normalized to $k_{0}$ are overlapped. (c) Close-up on the permittivity perturbation for the first sheet at the caustic, corresponding to the black square in (a). Colored circles represent vertices of the 3-D tetrahedral mesh in the $z=0$ plane. In (a,b,c), mesh resolution is $N_{r} \times N_{\theta} \times N_{y}=151 \times 181 \times 5$ with $m=2$. (d) Scaling of total CPU time as a function of energy conservation error expressed as a percentage of the total exchanged energy. Error scaling without AMR steps and as a function of $N_{r}$ is shown as a dashed black line. Simulations with $m=0, m=1$ and $m=2$ are shown with circles, triangles and squares, respectively. Each color represents a unique value of $N_{r}$, indicated with text in the figure.

tion and clamping local gains accordingly. Finally, it is worth mentioning here that there is no factor applied to caustic fields; these are all reconstructed intrinsically using the Etalon Integral method. The model does not have any free parameters.

\section{B. Energy transfer at caustic between two beams}

We now add a second beam incident at $90^{\circ}$ relative to the first such that the CBET interaction occurs between four wavefields (see Fig. 1 (d)). The incident vacuum intensity of each beam is $2 \times 10^{15} \mathrm{~W} / \mathrm{cm}^{2}$. For simplicity, the beams are $s$-polarized. A direct comparisons of fields is given in Figs. $8(a, b)$. The beam overlap region is not compared, since it would require keeping all interference terms before summing intensities in IFRIIT, which is not implemented. Outside of that region, both codes agree to within $5 \%$ of the input field amplitude in most of the plasma. A larger $5-15 \%$ disagreement is seen in the narrow region between the caustic and the beam overlap region, which may be due to the absence of fields modeled in the caustic shadow in the IFRIIT case. This test case was also studied in Ref. 28.

A comparison of LPSE and IFRIIT that is particularly sensitive to the modeling as a whole can be obtained by studying the fields in the backscattered direction of one beam (e.g. the rightward beam), behind the field injectors (i.e. without the contribution from the input beam). This region combines: (i) light from the rightward beam reflected from the caustic, self-amplified by the incident light field (as shown in Sec. VII A) and (ii) depleted by highly-refracted side-scatter from the upward beam, and (iii) direct side-scatter of the upward beam, highlyrefracted from the caustic. The latter side-scatter of the upward beam is itself amplified by the rightward beam 
(a)

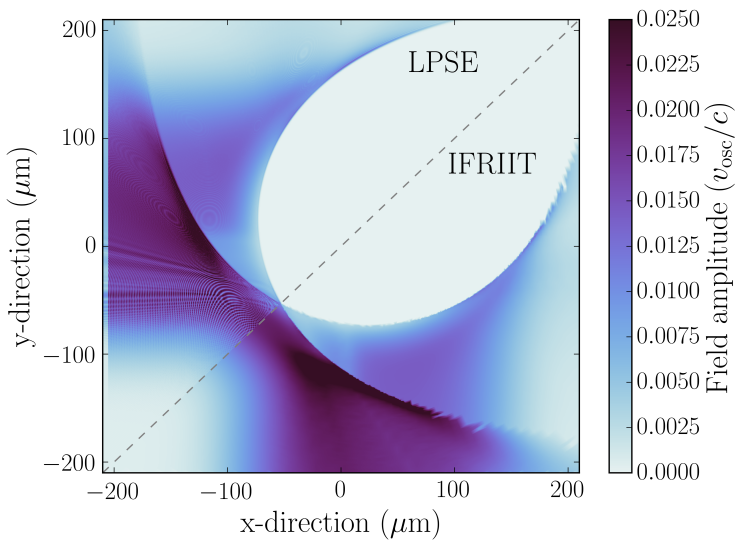

(c)

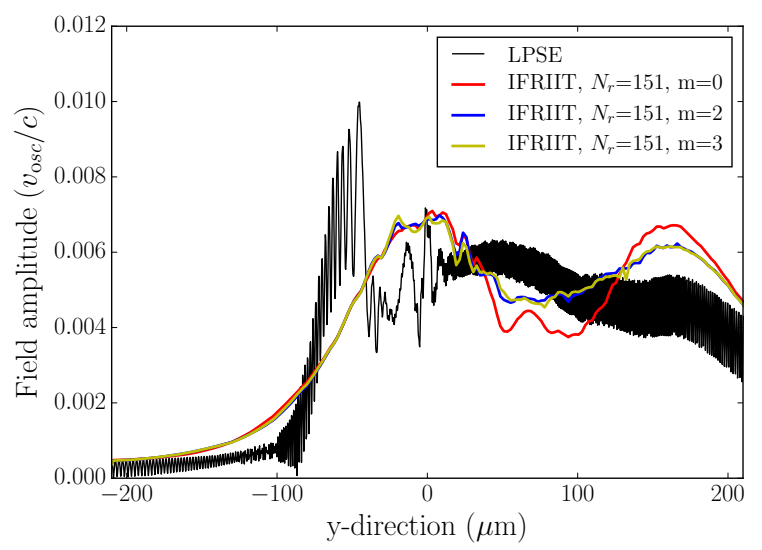

(b)

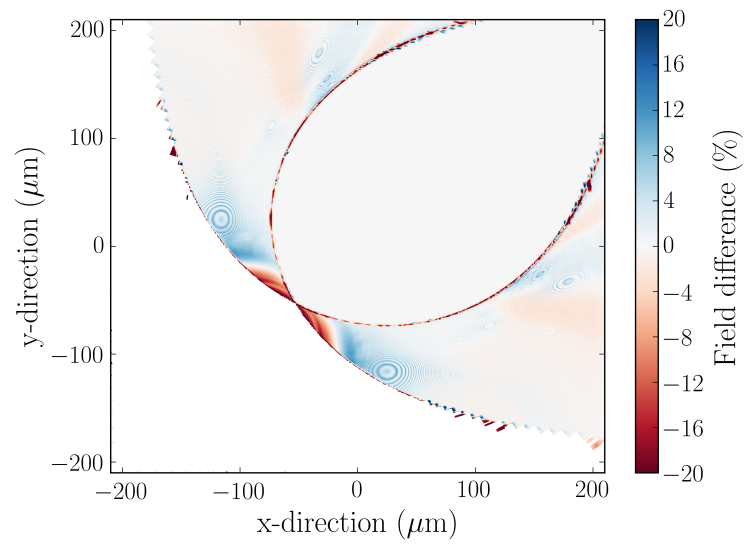

(d)

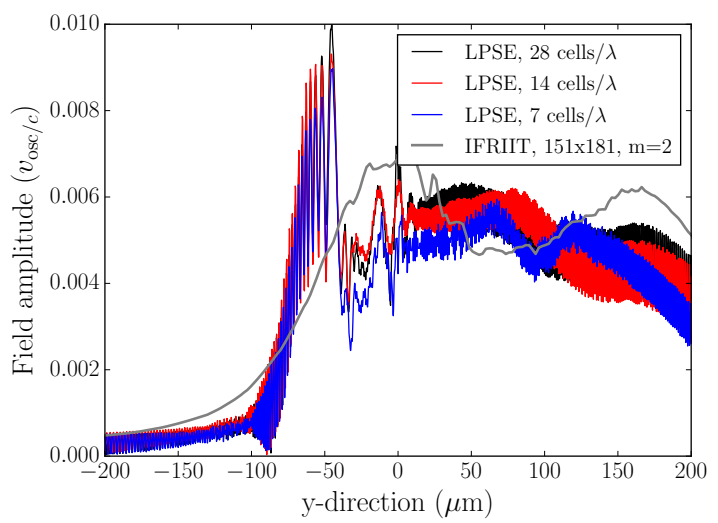

FIG. 8. (a) Fields in the 2-D simulation domain, as computed from LPSE (top-left quadrant) and IFRIIT (bottom-right quadrant). IFRIIT uses $181 \times 151 \times 5$ cells with level 2 refinement. LPSE uses $36000^{2}$ cells for the light solver (about 28 cells per wavelength), up-sampled by a factor of 2 for the IAW solver. To facilitate the comparison, the LPSE fields have been down-sampled by a factor of 16 and a maximum-filter of $3 \times 3$ pixel size has been applied. (b) Difference between the $L P S E$ and IFRIIT fields in 2-D, computed as $\left(\left|u_{\text {IFRIIT }}\right|-\left|u_{\text {LPSE }}\right|\right) /\left|u_{0}\right|$ where the input field $\left|u_{0}\right|=1.34 \times 10^{-2}$ is expressed in units of $v_{\text {osc }} / c$. The region of beam overlap in LPSE cannot be compared to IFRIIT without keeping the interference term ${ }^{\text {a }}$, and has been blanked. (c) Field profiles at $x=-210 \mu \mathrm{m}$, behind the first beam injectors for LPSE. Results from LPSE are shown with a black line. Results from IFRIIT using $N_{r}=151, N_{\theta}=181$ and $m \in[0,2,3]$ are shown with red, blue and yellow lines, respectively. (d) Field profiles at $x=-210 \mu \mathrm{m}$ for various LPSE resolutions; 28, 14 and 7 cells per wavelength, shown in black, red and blue, respectively. All LPSE simulation use a factor of 2 up-sampling for the IAWs. The IFRIIT solution using $151 \times 181$ with $\mathrm{m}=2$ is shown as a grey plain line.

${ }^{a}$ While this is possible to do in the framework of the model, IFRIIT is currently not formulated to keep the full interference term.

and depleted by its own backscatter. Such a comparison is provided in Fig. 8 (c). Despite the challenging modeling configuration, broad overall agreement is found. However, the structure of the scattered field does differ. A peak in scattered field is observed at $y=-75 \mu \mathrm{m}$ in LPSE, and is thought to be a diffraction-like feature. As such, it is not captured by the GO-driver in IFRIIT. The two other peaks observed in the scattered field differ between LPSE and IFRIIT in their location, but reach similar amplitude. This discrepancy may be related to several parameters already evoked in the previous section: lack of IAW advection in IFRIIT or the difficulty to reach convergence of the CBET calculation in LPSE. In particular, it is not clear that full convergence has been reached for the latter, as illustrated in Fig. 8 (d). Finally, it is worth mentioning that the LPSE calculation required about $300 \mathrm{k}$ CPU hours, versus about 3000 CPU seconds for the IFRIIT calculation. The level of agreement between the codes in this challenging configuration, especially in the plasma where energy deposition is occurring, remains satisfying.

\section{CONCLUSION}

We have described an implementation of Cross Beam Energy Transfer (CBET) within the framework of the IFRIIT code, an Inverse Ray-Tracing based model that 
reconstruct the phase and amplitude of wavefields in plasma, including at caustics, and with inline-like performance properties. The light propagation and CBET is described within a 3-D unstructured tetrahedron mesh that is adaptively refined to better resolve sub-grid-scale gradients of laser-related quantities, such as fields or permittivity perturbations. The modeling was applied to various academic test cases relevant to direct-drive and/or indirect-drive geometries. Solutions were compared and validated against the wave solver $L P S E$.

Various advantages of the modeling approach have been highlighted. First, the capability to describe permittivity perturbations on different ray sheets has been shown to be of importance in situations where a beam can interact with itself through CBET. Academic test cases in 1-D linear density profiles have shown consequent reductions in absorbed power of the order of $40 \%$ for short density scalelengths $(30 \mu \mathrm{m})$ and $13 \%$ for longer scalelengths $(300 \mu \mathrm{m})$, for moderate laser intensities of $5 \times 10^{14}$ $\mathrm{W} / \mathrm{cm}^{2}$. These conditions are relevant to the early laserplasma interaction, including for typical picket pulses employed in ICF.

Second, the interplay between CBET modeling and AMR has been demonstrated in a 1-D linear gain configuration modeled with 3-D top-hat beams. The interaction was described on an under-resolved mesh, typical of hydrodynamic codes for which the coronal plasma is loosely resolved. Calculations on such meshes showed that although the integrated CBET gain may be nearly correct (e.g. $10 \%$ below the reference), the detailed gain profile along the beam could significantly vary, here between $70 \%$ to $110 \%$ of the reference value. This is of importance for calculations where the detailed balance of the beam illumination is important, since the mesh itself may imprint gain perturbations onto the beam profiles. We have shown that the use of AMR is able to converge both the integrated gain and the local gain along the beam profile toward the reference value. Furthermore, we demonstrated how increasing the AMR depth controls energy conservation. The latter property is an interesting way to ensure energy conservation in more complex calculations without using non-physical methods such as re-normalizations of beam energy.

Last, we presented calculations of CBET in typical direct-drive geometries, which require describing (i) the separate couplings of incident and reflected wavefields, (ii) the caustic fields, and (iii) the sharp CBET gain boundary between the lit and shadow region at the caustic. These calculations were conducted using one and two beams at high intensities, $2-3 \times 10^{15} \mathrm{~W} / \mathrm{cm}^{2}$. Combining the multi-sheeted CBET approach, Etalon Integral and AMR methods in IFRIIT, we have shown the good agreement between IFRIIT and LPSE in both cases. While this highlights again the ability to control energy conservation through AMR, it also demonstrates the correct interplay of CBET with caustic fields reconstructed through an Etalon Integral. Excellent agreement was found in terms of total scattered power. In the single beam case (two-wavefields interaction), both codes agree to within $\simeq 5 \%$ of the input field. In the two-beams case (four-wavefields interaction), the agreement level lies in the $\simeq 5-15 \%$ range, depending on the region. Given the complexity of the test cases, it is expected that LPSE may not be entirely converged, although this may also highlight the limits of validity of the assumptions made in IFRIIT.

We have demonstrated the ability of the IFRIIT model to reproduce $\mathrm{CBET}$ in challenging configurations. We demonstrated the ability to (i) control energy conservation purely through adaptive meshing, (ii) reproduce $L P S E$ results with high accuracy while still describing the permittivity profile using a piece-wise linear sub-grid approximation and (iii) doing so without making assumptions on the caustic configuration other than it being of Fold type. Additionally, these results were obtained in computation times that are relevant for an inline implementation in a 3-D parallelized hydrocode. Finally, the correct CBET interaction, including at high gains, was obtained without using any numerical artifices, e.g. no limiters, multipliers, or energy re-normalization factors.

The successful comparisons between LPSE and IFRIIT validates the approximations made in the latter model for the laser-plasma interaction parameters considered here. Notably, in the present CBET formulation, the effects of phase delay and acceleration on the real part of the density perturbation, as well as complex refraction on gain gradients, were neglected. In addition, the model assumed steady state and neglected advection of the IAWs. While these assumptions were found to hold here, many other processes were left out of the comparison: polarization rotation, effects of speckles, strong IAW non-linearity and saturation, ponderomotive selffocusing, etc... While some of these effects did not play a role here, and other were not considered in the test cases, they are relevant to ICF and are considered as future work.

\section{ACKNOWLEDGMENTS}

This material is partially based upon work supported by the Department of Energy National Nuclear Security Administration under Award Number DE-NA0003856, the University of Rochester, and the New York State Energy Research and Development Authority.

This report was prepared partially as an account of work sponsored by an agency of the U.S. Government. Neither the U.S. Government nor any agency thereof, nor any of their employees, makes any warranty, express or implied, or assumes any legal liability or responsibility for the accuracy, completeness, or usefulness of any information, apparatus, product, or process disclosed, or represents that its use would not infringe privately owned rights. Reference herein to any specific commercial product, process, or service by trade name, trademark, manufacturer, or otherwise does not necessarily constitute or 
imply its endorsement, recommendation, or favoring by the U.S. Government or any agency thereof. The views and opinions of authors expressed herein do not necessarily state or reflect those of the U.S. Government or any agency thereof.

This work has been partially carried out within the framework of the EUROfusion Consortium and has received funding from the Euratom research and training program 2019-2020 under grant agreement No. 633053. The views and opinions expressed herein do not necessarily reflect those of the European Commission.

\section{Appendix A: Symmetric gradient conserving adaptive laser mesh refinement}

Introducing new IRT observation points in the mesh allows one to reconstruct non-linear subgrid variations of laser-related quantities. For these subgrid variations to impact the phase and amplitude of rays, the added points must be part of the tetrahedral mesh itself. This can be achieved by refining tetrahedra into sub-tetrahedra, in which gradients of laser-related quantities are obtained through IRT at the newly added nodes. Effectively, AMR coupled to IRT allows one to gradually resolve laserrelated fine-scale structures until the piecewise linear subgrid approximation recovers its accuracy.

\section{Refinement strategy}

Refinement of the laser mesh impacts subsequent laserrelated computations if it modifies the dielectric permittivity seen by each wavefield. Namely, both ray trajectories and phase computations may be affected, which in turns warrant iteration of the IRT step on the whole mesh. This is because a localized modification of $\epsilon$ may impact larger regions of space if rays traverse it. Given the potentially high associated numerical cost, we work in a simplified framework where the AMR steps are required to leave ray trajectories unmodified. This implies that both ray parameters and ray amplitudes are unchanged by AMR steps. Mathematically, the updated permittivity profile must be considered as a perturbation term from the background permittivity, similarly to how $\operatorname{Im}(\epsilon)$ is treated in the GO framework. While this may be implemented within CGO algorithms, this approach is better suited to the real-valued GO framework, where trajectories are only affected by $\operatorname{Re}(\epsilon)$. For this reason, this work focuses on the GO-framework implemented in IFRIIT. While the IRT step must still be computed on the vertices of the new tetrahedra, finding the associated ray parameters is straightforward since those of the parent tetrahedron are known in advance, which allows for an excellent IRT initial guess for efficient convergence.

The laser mesh refinement strategy we propose follows three principles: (i) the new tetrahedra formed by adding verticies do not modify the user-supplied gradi-

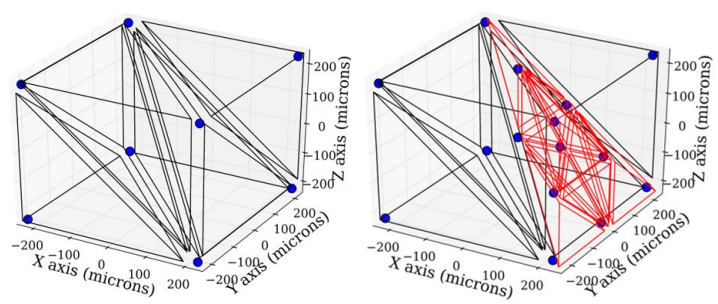

FIG. 9. Illustration of a tetrahedron being refined by one level. The point distribution is shown with blue dots, and the edges of the initial triangulation are shown with black lines. The volumes of the tetrahedra is reduced for easier visualization. (a) Initial point distribution and tetrahedra. (b) Refined tetrahedra (red) obtained after insertion of 7 nodes.

ents in hydrodynamic quantities, (ii) the algorithm can be applied recursively and (iii) the algorithm does not introduce preferential directions. The first condition ensures that laser refraction is not modified by inserting new tetrahedra, since it depends only on $\operatorname{Re}(\epsilon)$ in the GO framework and in absence of LPIs. The second condition allows iterative AMR steps in order to meet a convergence criterion. The last condition is intended to avoid introduction of directional bias through AMR. All these conditions are obtained by splitting parent tetrahedra into sub-tetrahedra, as detailed in the next section.

\section{Tetrahedron splitting}

Consider a generic tetrahedron consisting of 4 vertices, 6 edges and 4 faces. We insert a point at the center of each edge, splitting the tetrahedron volume into 4 subtetrahedra and an octahedron. The octahedron can be most efficiently split into 4 sub-tetrahedra, but this involves the choice of a preferential diagonal. As such, one more point is inserted at the octahedron center, thus splitting it into 8 sub-tetrahedra. The main difficulty in the splitting algorithm then arises in reconnecting subfaces within the same refined parent tetrahedron, but also with neighborhood cells that may or may not have been refined, potentially to a different refinement depth. In that context, the algorithm in IFRIIT does not allow for more than 4 sub-faces to be present on a face, which in turn forces adjacent tetrahedra to be refined when iteratively refining the mesh. One-pass refinement of one tetrahedron in a example mesh is illustrated in Fig. 9.

\section{Splitting criterion}

A tetrahedron is chosen to be split when it is estimated that the linear distribution of a quantity $F$ is not representative of the real sub-grid distribution. We have chosen a simple criterion based on the comparison between a maximum rate of change of $F$ to the average value in the cell $\langle|F|\rangle$. The maximum rate of change 
is computed by multiplying each component of the linear gradient $|\nabla F|$ with each component of the maximum distance of the cell vertices to the barycenter. Additionally, it is required that the maximum value of $F$ in the cell is large enough compared to the maximum value in the entire mesh. The criterion reads:

$$
\begin{array}{r}
\max \left(|\nabla F| \circ \max _{i \in \operatorname{cell}}\left(\left|\mathbf{r}_{i}-\mathbf{r}_{\mathrm{b}}\right|\right)\right)>N\langle|F|\rangle, \\
\&\langle|F|\rangle>\max _{j \in \operatorname{mesh}}\left(\left|F_{j}\right|\right) / 50,
\end{array}
$$

where the $\mathbf{r}_{i}$ are the coordinates of the tetrahedron vertices, $\mathbf{r}_{\mathrm{b}}$ is the tetrahedron's barycenter, o denotes an element-wise product (Hadamard product) and $N$ is a constant used to set the sensitivity of the refiner algorithm to gradients. All calculations presented in this paper use $N=1$. Note that cells that share a vertex with the simulation domain boundary are excluded.

\section{Appendix B: IAW response models used in IFRIIT}

The density perturbation term implemented in CBET codes is usually described by either linear fluid or linear kinetic models. IFRIIT implements both approaches, for which we give a summary of the mathematical formulation in this appendix.

\section{Kinetic formulation}

The linear kinetic model is a $k$-space dependent formulation of the Landau IAW damping rate. It is the preferred formulation for use in IFRIIT, as it allows one to account for both the fast and slow modes of mixed species plasmas. Assuming slowly varying envelopes for the ponderomotive and electrostatic potentials, the amplitude of the density perturbation is obtained by linearization of the Vlasov equation ${ }^{16,26}$. The perturbation $\delta n_{m n}$ seen by a wavefield $m$ of wavevector $\mathbf{k}_{m}$ (propagating in sheet $\Omega_{s}^{b}$ ), overlapping with a wavefield $n$ of wavevector $\mathbf{k}_{n}$ and field amplitude $a_{n}$ (propagating in sheet $\Omega_{\hat{s}}^{\hat{b}}$ ), reads:

$$
\frac{\delta n_{m n}}{n_{e}}=\frac{1}{4} K_{m n}^{*} \frac{k_{s}^{2} c^{2}}{\omega_{p e}^{2}}\left|a_{n}\right|^{2}
$$

where $K_{m n}=\chi_{e}\left(1+\chi_{i}\right) /\left(1+\chi_{e}+\chi_{i}\right)$ characterizes the plasma response, and the density perturbation is written for aligned polarizations. The electron and ion susceptibilities $\chi_{e}$ and $\chi_{i}$ read, assuming thermodynamic equilibrium:

$$
\begin{aligned}
& \chi_{e}=-\frac{1}{2 k_{s}^{2} \lambda_{D e}^{2}} Z^{\prime}\left(\frac{\bar{\omega}}{k_{s} v_{T e} \sqrt{2}}\right) \\
& \chi_{i}=-\frac{1}{2 k_{s}^{2} \lambda_{D e}^{2}} \frac{T_{e}}{\langle Z\rangle} \sum_{\alpha}^{\text {species }} \frac{f_{\alpha} Z_{\alpha}^{2}}{T_{\alpha}} Z^{\prime}\left(\frac{\bar{\omega}}{k_{s} v_{T \alpha} \sqrt{2}}\right),
\end{aligned}
$$

with $\mathbf{k}_{s}=\mathbf{k}_{m}-\mathbf{k}_{n}$ the grating wavevector, $k_{s}=\left|\mathbf{k}_{s}\right|$, $\omega_{\text {beat }}=\omega_{m}-\omega_{n}$ the beat wave frequency, $\mathbf{u}$ the flow velocity vector, $\bar{\omega}=\omega_{\text {beat }}-\mathbf{k}_{s}$. $\mathbf{u}$ the effective frequency in the plasma rest frame, $Z^{\prime}$ the derivative of the plasma dispersion function, $v_{T \alpha}=\sqrt{k_{\mathrm{B}} T_{\alpha} / m_{\alpha}}$ the thermal velocity of species $\alpha$ with temperature $T_{\alpha}$ and mass $m_{\alpha}$, $\lambda_{D e}=\sqrt{\epsilon_{0} k_{\mathrm{B}} T_{e} / n_{e} e^{2}}$ is the electron Debye length, $Z_{\alpha}$ is the ionization of species $\alpha$ with fraction $f_{\alpha}$, and $\langle Z\rangle=\sum_{\alpha} f_{\alpha} Z_{\alpha}$ is the average ionization.

\section{Fluid formulation}

In the fluid approach, the linearized ponderomotive modification of the plasma density due to the beating of two laser waves is obtained by Fourier transforms of the linearized fluid equations. By taking the Fourier variables to be $\bar{\omega}$ and $\mathbf{k}_{s}{ }^{1,30}$, the perturbation $\delta n_{m n}$ seen by a wavefield $m$ of wavevector $\mathbf{k}_{m}$ (propagating in sheet $\Omega_{s}^{b}$ ), overlapping with a wavefield $n$ of wavevector $\mathbf{k}_{n}$ and field amplitude $a_{n}$ (propagating in sheet $\Omega_{\hat{s}}^{\hat{b}}$ ), reads:

$$
\begin{aligned}
\frac{\delta n_{m n}}{n_{e}} & =\frac{c^{2}}{4 v_{T e}^{2}} \frac{\left(k_{s} C_{s e}\right)^{2}}{1+k_{s}^{2} \lambda_{D e}^{2}}\left(\frac{1}{\mathcal{D}_{s}}\right)\left|a_{n}\right|^{2} \\
\mathcal{D}_{s} & =\bar{\omega}^{2}+2 \imath \bar{\omega} \nu_{s}-\omega_{s}^{2} \\
\omega_{s}^{2} & =k_{s}^{2}\left(\frac{C_{s e}^{2}}{1+k_{s}^{2} \lambda_{D e}^{2}}+3 v_{T i}^{2}\right) \\
C_{s e}^{2} & =\langle Z\rangle k_{\mathrm{B}} T_{e} / m_{i}
\end{aligned}
$$

where $\omega_{s}$ is the grating frequency, $\mathcal{D}_{s}$ is the resonance denominator, and $\nu_{s}$ is an IAW damping rate, which must be prescribed.

${ }^{1}$ D. Pesme, G. Bonnaud, M. Casanova, R. Dautray, C. Labaune, G. Laval, L. Bergé, and J.P. Watteau. La fusion thermonucléaire inertielle par laser : l'interaction laser-matière part. 1, vol. 1. (French Edition). Synthèses. Eyrolles, 61, Boulevard SaintGermain, 1993.

${ }^{2} \mathrm{~S}$. Atzeni and J. Meyer-ter Vehn. The Physics of Inertial Fusion. International Series of Monographs on Physics. Oxford University Press, Oxford, 2004.

${ }^{3}$ P. Michel, L. Divol, E. A. Williams, C. A. Thomas, D. A. Callahan, S. Weber, S. W. Haan, J. D. Salmonson, N. B. Meezan, O. L. Landen, S. Dixit, D. E. Hinkel, M. J. Edwards, B. J. MacGowan, J. D. Lindl, S. H. Glenzer, and L. J. Suter. Energy transfer between laser beams crossing in ignition hohlraums. Physics of Plasmas, 16(4), 2009.

${ }^{4}$ I. V. Igumenshchev, W. Seka, D. H. Edgell, D. T. Michel, D. H. Froula, V. N. Goncharov, R. S. Craxton, L. Divol, R. Epstein, R. Follett, J. H. Kelly, T. Z. Kosc, A. V. Maximov, R. L. McCrory, D. D. Meyerhofer, P. Michel, J. F. Myatt, T. C. Sangster, A. Shvydky, S. Skupsky, and C. Stoeckl. "crossed-beam energy transfer in direct-drive implosions". Physics of Plasmas, 19(5):056314, May 2012.

${ }^{5}$ A. Colaïtis, G. Duchateau, X. Ribeyre, and V. Tikhonchuk. Modeling of the cross-beam energy transfer with realistic inertialconfinement-fusion beams in a large-scale hydrocode. Phys. Rev. E, 91:013102, January 2015.

${ }^{6}$ D. J. Strozzi, D. S. Bailey, P. Michel, L. Divol, S. M. Sepke, G. D. Kerbel, C. A. Thomas, J. E. Ralph, J. D. Moody, and 
M. B. Schneider. Interplay of laser-plasma interactions and inertial fusion hydrodynamics. Phys. Rev. Lett., 118:025002, Jan 2017.

${ }^{7}$ S. Gus'kov, X. Ribeyre, M. Touati, J.-L. Feugeas, P. Nicolaï, and V. Tikhonchuk. "ablation pressure driven by an energetic electron beam in a dense plasma". Physical Review Letters, 109(25):255004, December 2012.

${ }^{8}$ A. Colaïtis, X. Ribeyre, E. Le Bel, G. Duchateau, Ph. Nicolaï, and V. Tikhonchuk. Influence of laser induced hot electrons on the threshold for shock ignition of fusion reactions. Physics of Plasmas, 23(7):072703, 2016.

${ }^{9}$ E. Llor Aisa, X. Ribeyre, G. Duchateau, T. Nguyen-Bui, V. T. Tikhonchuk, A. Colaïtis, R. Betti, A. Bose, and W. Theobald. The role of hot electrons in the dynamics of a laser-driven strong converging shock. Physics of Plasmas, 24(11):112711, 2017.

${ }^{10}$ R. Nora, W. Theobald, R. Betti, F.J. Marshall, D.T. Michel, W. Seka, B. Yaakobi, M. Lafon, C. Stoeckl, J. Delettrez, A.A. Solodov, A. Casner, C. Reverdin, X. Ribeyre, A. Vallet, J. Peebles, F.N. Beg, and M.S. Wei. Gigabar spherical shock generation on the omega laser. Phys. Rev. Lett., 114:045001, January 2015.

${ }^{11}$ A. Colaïtis, G. Duchateau, X. Ribeyre, Y. Maheut, G. Boutoux, L. Antonelli, Ph. Nicolaï, D. Batani, and V. Tikhonchuk. Coupled hydrodynamic model for laser-plasma interaction and hot electron generation. Phys. Rev. E, 92:041101, October 2015.

${ }^{12}$ D. Turnbull, A. Colaitis, R. K. Follett, J. P. Palastro, D. H. Froula, P. Michel, C. Goyon, T. Chapman, L. Divol, G. E. Kemp, D. Mariscal, S. Patankar, B. B. Pollock, J. S. Ross, J. D. Moody, E. R. Tubman, and N. C. Woolsey. Crossed-beam energy transfer: polarization effects and evidence of saturation. Plasma Physics and Controlled Fusion, 60(5), April 2018.

${ }^{13}$ D. Turnbull, A. Colaïtis, A. M. Hansen, A. L. Milder, J. P. Palastro, J. Katz, C. Dorrer, B. E. Kruschwitz, D. J. Strozzi 2, and D. H. Froula. Impact of the langdon effect on crossed-beam energy transfer. Submitted to Nature Physics.

${ }^{14}$ I. V. Igumenshchev, D. H. Edgell, V. N. Goncharov, J. A. Delettrez, A. V. Maximov, J. F. Myatt, W. Seka, A. Shvydky, S. Skupsky, and C. Stoeckl. "crossed-beam energy transfer in implosion experiments on omega". Physics of Plasmas, 17(12):122708, December 2010.

${ }^{15}$ J. A. Marozas and T. J. B. Collins. "cross-beam energy transfer (cbet) effect with additional ion heating integrated into the 2-d hydrodynamics code draco". In APS Meeting Abstracts, page 5003, October 2012.

${ }^{16}$ P. Michel, L. Divol, E. A. Williams, S. Weber, C. A. Thomas, D. A. Callahan, S. W. Haan, J. D. Salmonson, S. Dixit, D. E. Hinkel, M. J. Edwards, B. J. MacGowan, J. D. Lindl, S. H. Glenzer, and L. J. Suter. "tuning the implosion symmetry of icf targets via controlled crossed-beam energy transfer". Physical Review Letters, 102(2):025004, January 2009.

${ }^{17}$ A. Colatis, T. Chapman, D. Strozzi, L. Divol, and P. Michel. A tesselation-based model for intensity estimation and laser plasma interactions calculations in three dimensions. Physics of Plasmas, 25(3):033114, 2018.

${ }^{18}$ P. Michel, L. Divol, R. P. J. Town, M. D. Rosen, D. A. Callahan, N. B. Meezan, M. B. Schneider, G. A. Kyrala, J. D. Moody, E. L. Dewald, K. Widmann, E. Bond, J. L. Kline, C. A. Thomas, S. Dixit, E. A. Williams, D. E. Hinkel, R. L. Berger, O. L. Landen, M. J. Edwards, B. J. MacGowan, J. D. Lindl, C. Haynam, L. J. Suter, S. H. Glenzer, and E. Moses. Three-wavelength scheme to optimize hohlraum coupling on the national ignition facility. Phys. Rev. E, 83:046409, Apr 2011.

${ }^{19}$ R. P. J. Town, M. D. Rosen, P. A. Michel, L. Divol, J. D. Moody, G. A. Kyrala, M. B. Schneider, J. L. Kline, C. A. Thomas, J. L. Milovich, D. A. Callahan, N. B. Meezan, D. E. Hinkel, E. A. Williams, R. L. Berger, M. J. Edwards, L. J. Suter, S. W. Haan, J. D. Lindl, E. L. Dewald, S. Dixit, S. H. Glenzer, O. L. Landen, E. I. Moses, H. A. Scott, J. A. Harte, and G. B. Zimmerman. Analysis of the national ignition facility ignition hohlraum energetics experiments. Physics of Plasmas, 18(5):056302, 2011.

${ }^{20}$ A. K. Davis, D. Cao, D. T. Michel, M. Hohenberger, D. H. Edgell, R. Epstein, V. N. Goncharov, S. X. Hu, I. V. Igumenshchev, J. A. Marozas, A. V. Maximov, J. F. Myatt, P. B. Radha, S. P. Regan, T. C. Sangster, and D. H. Froula. Isolating and quantifying crossbeam energy transfer in direct-drive implosions on omega and the national ignition facility. Physics of Plasmas, 23(5):056306, 2016.

${ }^{21}$ A. Colatis, J. P. Palastro, R. K. Follett, I. V. Igumenschev, and V. Goncharov. Real and complex valued geometrical optics inverse ray-tracing for inline field calculations. Physics of Plasmas, 26(3):032301, 2019.

${ }^{22}$ J. F. Myatt, R. K. Follett, J. G. Shaw, D. H. Edgell, D. H. Froula, I. V. Igumenshchev, and V. N. Goncharov. A wave-based model for cross-beam energy transfer in direct-drive inertial confinement fusion. Physics of Plasmas, 24(5):056308, 2017.

${ }^{23}$ R. K. Follett, D. H. Edgell, D. H. Froula, V. N. Goncharov, I. V. Igumenshchev, J. G. Shaw, and J. F. Myatt. Full-wave and ray-based modeling of cross-beam energy transfer between laser beams with distributed phase plates and polarization smoothing. Physics of Plasmas, 24(10):103128, 2017.

${ }^{24}$ T. Dewandre, J. R. Albritton, and E. A. Williams. Doppler shift of laser light reflected from expanding plasmas. Physics of Fluids, 24:528-536, March 1981.

${ }^{25}$ A. Debayle, C. Ruyer, O. Morice, P.-E. Masson-Laborde, P. Loiseau, and D. Benisti. A unified modeling of wave mixing processes with the ray tracing method. Submitted to Physics of Plasmas.

${ }^{26}$ P. Michel, L. Divol, D. Turnbull, and J. D. Moody. Dynamic control of the polarization of intense laser beams via optical wave mixing in plasmas. Phys. Rev. Lett., 113:205001, November 2014.

${ }^{27}$ P. Michel, W. Rozmus, E. A. Williams, L. Divol, R. L. Berger, S. H. Glenzer, and D. A. Callahan. Saturation of multi-laser beams laser-plasma instabilities from stochastic ion heating. Physics of Plasmas, 20(5):056308, 2013.

${ }^{28}$ R. K. Follett, J. G. Shaw, J. F. Myatt, V. N. Goncharov, D. H. Edgell, D. H. Froula, and J. P. Palastro. Ray-based modeling of cross-beam energy transfer at caustics. Phys. Rev. E, 98:043202, Oct 2018 .

${ }^{29}$ Y. A. Kravtsov and N. Y. Zhu. Theory of Diffraction, Heuristic Approaches. Alpha Science Series on Wave Phenomena. Alpha Science International LTD., Oxford, U.K., 2010.

${ }^{30}$ A. Colaïtis, S. Hüller, D. Pesme, G. Duchateau, and V. T. Tikhonchuk. Crossed beam energy transfer: Assessment of the paraxial complex geometrical optics approach versus a timedependent paraxial method to describe experimental results. Physics of Plasmas, 23(3):032118, 2016.

${ }^{31}$ Note that this definition is inaccurate in the case of CGO in dissipative media, where caustics may be shifted in complex space.

${ }^{32}$ IFRIIT is fully formulated in 3-D and the AMR step cannot be constrained to a plane 